\title{
Accretion and ejection properties of embedded protostars: the case of HH26, HH34, and HH46 IRS ${ }^{\star}$
}

\author{
S. Antoniucci ${ }^{1,2}$, B. Nisini ${ }^{2}$, T. Giannini ${ }^{2}$, and D. Lorenzetti ${ }^{2}$ \\ 1 Università degli Studi di Roma “Tor Vergata”, via della Ricerca Scientifica 1, 00133 Roma, Italy \\ e-mail: antoniucci@oa-roma.inaf.it \\ 2 INAF-Osservatorio Astronomico di Roma, via di Frascati 33, 00040 Monteporzio Catone, Italy
}

Received 13 March 2007 / Accepted 25 October 2007

\section{ABSTRACT}

\begin{abstract}
Aims. We present the results of a spectroscopic analysis of three young embedded sources (HH26 IRS, HH34 IRS, and HH46 IRS) belonging to different star-forming regions and displaying well-developed jet structures. The aim is to investigate the source accretion and ejection properties and their connection.

Methods. We used VLT-ISAAC near-IR medium resolution $(R \sim 9000)$ spectra ( $H$ and $K$ bands) to derive, in a self-consistent way, parameters like the star luminosity, accretion luminosity, and the mass accretion rate. Mass ejection rates have also been estimated from analysing different emission features.

Results. The spectra present several emission lines but no photospheric features in absorption, indicating a large veiling in both the $H$ and $K$ bands. In addition to features commonly observed in jet driving sources ([Fe II], $\mathrm{H}_{2}, \mathrm{H} \mathrm{I}, \mathrm{CO}$ ), we detect a number of emission lines due to permitted atomic transitions, such as $\mathrm{Na}$ I and Ti I that are only 2-5 times weaker than the Br $\gamma$ line. Some of these features remain unidentified. Emission from Na I $2.2 \mu \mathrm{m}$ doublet is observed along with $\mathrm{CO}(2-0)$ band-head emission, indicating a common origin in an inner gaseous disc heated by accretion. We find that accretion provides about $50 \%$ and $80 \%$ of the bolometric luminosity in HH26 IRS and HH34 IRS, as expected for accreting young objects. Mass accretion and loss rates spanning $10^{-6}-10^{-8} M_{\odot} \mathrm{yr}^{-1}$ have been measured. The derived $\dot{M}_{\text {loss }} / \dot{M}_{\text {acc }}$ is $\sim 0.01$ for HH26 IRS and HH34 IRS, and $>0.1$ for HH46 IRS. These numbers are in the range of values predicted by MHD jet launching models and found in the most active classical T Tauri stars.

Conclusions. Comparison with other spectroscopic studies performed on Class Is seems to indicate that Class Is actually having accretion-dominated luminosities are limited in number. Although the analysed sample is small, we can tentatively define some criteria to characterise such sources: they have $K$-band veiling larger than 2 and, in the majority of the cases, present IR features of $\mathrm{CO}$ and $\mathrm{NaI}$ in emission, although these do not directly correlate with the accretion luminosity. Class Is with massive jets have high $L_{\text {acc }} / L_{\text {bol }}$ ratios but not all the identified accretion-dominated objects present a jet. As suggested by the SEDs of our three objects, the accretion-dominated objects could be in an evolutionary transition phase between Class 0 and I. Studies of the kind presented here, but on larger samples of possible candidates, should be performed in order to test and refine these criteria.
\end{abstract}

Key words. stars: formation - stars: evolution - infrared: stars - techniques: spectroscopic

\section{Introduction}

The process of mass accretion accompanying the formation of solar type stars is always associated with mass ejection in the form of collimated jets that extend from few AU up to parsec distances from the exciting source. According to an established class of models (Königl \& Pudritz 2000; Casse \& Ferreira 2000), accretion and ejection are indeed intimately related through the presence of a magnetised accretion disc: the jets carry away the excess angular momentum, so that part of the disc material can move toward the star. The efficiency of this process is measured by the ratio between the mass ejection and mass accretion rates and depends on the jet acceleration mechanism at work. Measurements of such an efficiency have so far been only obtained for classical T Tauri stars, whose accretion properties are fairly well-studied through observations of the excess continuum emission at optical and UV wavelengths. Values of $\dot{M}_{\text {loss }} / \dot{M}_{\text {acc }}$ in the range $1-10 \%$ have been found by different studies (e.g. Hartigan et al. 1995; Woitas et al. 2005; Ferreira et al. 2006). It is

\footnotetext{
* Based on observations collected at the European Southern Observatory, Chile (ESO Programmes 072.C-0176(A), 074.C0235(A)).
}

nevertheless important to test accretion/ejection models in young sources at earlier stages of evolution, when accretion dominates the energetics of the system, so that the mechanism to extract angular momentum is expected to be more efficient. To this aim, an interesting sample of objects are Class I sources, i.e. the class of embedded stars characterised by a steeply rising IR spectral energy distribution (SED) between 2 and $10 \mu \mathrm{m}$ and usually considered younger than visible T Tauri stars (the Class II sources). However, the high extinction pertaining to these objects strongly limits the measurement of their stellar and accretion properties that is needed to prove that they are indeed in a phase of higher accretion with respect to Class II sources. The general assumption so far applied has been that most of the bolometric luminosity of Class I objects is due to accretion.

Recently, however, thanks to the use of high dispersion sensitive instrumentation, it has become possible to define the stellar properties of small samples of Class I stars through their weak photospheric lines detected in the optical scattered light (White $\&$ Hillenbrand 2004) and in the near-IR direct emission (Greene \& Lada 2002; Nisini et al. 2005a; Doppmann et al. 2005). Such studies have shown that the characteristics of Class I objects in fact vary significantly. In particular, the accretion luminosity 
Table 1. The observed targets and their main observational properties.

\begin{tabular}{|c|c|c|c|c|c|c|c|c|c|c|}
\hline \multirow[t]{2}{*}{ Source } & \multirow[t]{2}{*}{$\mathrm{RA}(2000)$} & \multirow[t]{2}{*}{$\operatorname{Dec}(2000)$} & \multirow{2}{*}{$\begin{array}{c}m_{\mathrm{J}} \\
(\mathrm{mag}) \\
2 \mathrm{MASS}^{(1)}\end{array}$} & \multicolumn{2}{|c|}{$\begin{array}{c}m_{\mathrm{H}} \\
(\mathrm{mag})\end{array}$} & \multicolumn{2}{|c|}{$\begin{array}{c}m_{\mathrm{K}} \\
(\mathrm{mag})\end{array}$} & \multirow[t]{2}{*}{$\begin{array}{c}D \\
(\mathrm{pc})\end{array}$} & \multirow[t]{2}{*}{$\alpha^{a}$} & \multirow[t]{2}{*}{$\begin{array}{l}L_{\mathrm{bol}} \\
\left(L_{\odot}\right)\end{array}$} \\
\hline & & & & $2 \operatorname{MASS}^{(1)}$ & This work $^{b}$ & $2 \mathrm{MASS}^{(1)}$ & This work $^{b}$ & & & \\
\hline HH 26 IRS & 054603.9 & -001452 & 16.77 & 14.07 & 14.6 & 11.88 & 12.3 & 450 & 2.01 & $4.6-9.2$ \\
\hline HH 34 IRS & 053529.9 & -062658 & 15.06 & 13.60 & 13.5 & 12.38 & 12.4 & 460 & 1.14 & $12.4-19.9$ \\
\hline HH 46 IRS & 082543.9 & -510036 & 14.20 & 12.88 & 14.8 & 12.72 & 13.4 & 450 & 1.96 & $<15.0^{(c)}$ \\
\hline
\end{tabular}

Notes. ${ }^{a}$ The spectral index $\alpha=\mathrm{d} \log \left(\lambda F_{\lambda}\right) / \mathrm{d} \log (\lambda)$ is calculated between 2 and $10 \mu \mathrm{m} .{ }^{b}$ The magnitude values in the $H$ and $J$ bands have been estimated from the calibrated spectra. ${ }^{c}$ Total luminosity: the source is a binary (see text for details). References. (1) from the 2MASS catalogue (Skrutskie et al. 2006).

may span from a few percent up to $80 \%$ of the bolometric luminosity; these findings show that not all sources defined as Class I are indeed actively accreting objects and suggest that a classification based on different criteria is indeed required.

White \& Hillenbrand (2004), notably, derived from their analysis of the scattered light spectra of Taurus-Auriga sources that the average $\dot{M}_{\text {loss }} / \dot{M}_{\text {acc }}$ for Class I of their sample is larger than for Class II and close to unity. They interpret this result as due to an observational bias induced by the effect of disc orientation in their optical spectra; if the Class I objects are seen prevalently edge-on, then the extended region emitting the forbidden lines from which they estimate $\dot{M}_{\text {loss }}$ is seen more directly than the obscured stellar photosphere. It is clear that this kind of biases can be minimised by performing spectroscopic observations directly in the IR, where features originating in the photosphere, in the accretion region, and in the jet can be simultaneously detected by instrumentation that is sensitive enough.

In this framework, we report here the results of near-IR spectroscopic observations at medium resolution of three embedded sources (HH34 IRS, HH26 IRS, and HH46 IRS) displaying welldeveloped jet structures and having the spectral index between 2 and $10 \mu \mathrm{m}$ typical of Class I objects. We have derived accretion and ejection parameters of these sources through analysing the different features detected on the spectra. The goal is to study how much of their energy is due to accretion and to investigate the efficiency of the ejection mechanism.

We describe the sample and the observations in Sect. 2 and report the results in Sect. 3; in Sect. 4 we present the procedures applied for the analysis of the data and infer the physical properties of the objects and their jets. These results are then discussed in Sect. 5, where a comparison with similar sources analysed in previous studies will be also made. The main conclusions of our work are summarised in Sect. 6.

\section{Description of the sample and observations}

Our sample consists of three sources, namely HH26 IRS, HH34 IRS, and HH46 IRS, selected among sources having a positive spectral index between 2 and $10 \mu \mathrm{m}$ and showing welldefined jet structures observed in the near-IR very close to the source itself. The basic observational properties of the targets are listed in Table 1.

HH34 IRS (located in the L1641 cloud, at a distance of $460 \mathrm{pc}$ ) is the exciting source of the spectacular parsec-scale HH34 flow (Bally \& Devine 1994). It was initially detected as a faint optical star by Reipurth et al. (1986), but Reipurth et al. (2000) show that the optical emission is coming from a very compact reflection nebulosity, while the source itself is an embedded IR object displaced at about 0.1 arcsec from this nebulosity. HH34 IRS was detected by IRAS and at sub-mm/mm wavelengths by Reipurth et al. (1993) and Johnstone et al. (2006), who show that a dense cloud core surrounds the object. The HH34 jet has been studied in detail both in the optical (Reipurth et al. 2002; Bacciotti \& Eislöffel 1999) and in the IR (Podio et al. 2006; Takami et al. 2006; Garcia Lopez et al. 2007), where the $\mathrm{H}_{2}$ molecular counterpart of the atomic jet has been also detected. Jet proper motion analysis has shown that the jet is oriented with an angle ranging from 20 to $30^{\circ}$ to the plane of the sky (Eislöffel \& Mundt 1992; Heathcote \& Reipurth 1992).

HH26 IRS (located in the L1630 molecular cloud at a distance of $450 \mathrm{pc}$ ) is an embedded object, recognised to be the exciting source of the HH26A/C/D chain and of the associated molecular outflow by Davis et al. (1997). Davis et al. (2002) discovered that this source is also driving a small-scale $\mathrm{H}_{2}$ jet with a length of a few arcsec. An IR spectrum of HH26 IR from 2 to $4 \mu \mathrm{m}$ was obtained by Simon et al. (2004), showing emission line features, from $\mathrm{H}_{2}, \mathrm{HI}$, and $\mathrm{CO}$, typical of active young sources. Observations at sub-mm/mm wavelengths of this object were obtained by Lis et al. (1999) and Johnstone et al. (2001).

HH46 IRS is located in an isolated Bok globule close to the Gum nebula (at a distance of $450 \mathrm{pc}$ ). The object actually consists of an embedded young binary system of $0.26^{\prime \prime}$ separation (Reipurth et al. 2000) that illuminates a reflection nebula from which the HH46/47 system of Herbig Haro objects emerges. At infrared wavelengths, the redshifted counter-jet was detected penetrating through the reflection nebula down to the exciting source (Eislöffel et al. 1994). HH46-IRS is associated with the IRAS object IRAS 08242-5050 and was detected in the millimetre range by Reipurth et al. (1993). A spectrum at mid-IR wavelength of HH46-IRS has been recently obtained by Spitzer (Noriega-Crespo et al. 2004). Proper motion measurements of the HH46/47 objects indicate the HH46 IRS jet is inclined by $\sim 34^{\circ}$ to the plane of the sky (Eislöffel \& Mundt 1994).

The observations were performed with the VLT-UT1 ISAAC spectrograph in SW mode on 28, 29 and 30 December 2004. Three spectral regions were investigated: one in the $H$ band (1.57-1.65 $\mu \mathrm{m}$ for HH34 IRS and HH46 IRS and 1.61-1.69 $\mu \mathrm{m}$ for HH26 IRS), and two contiguous segments in the $K$ band $(2.08-2.30 \mu \mathrm{m})$. These spectral regions were chosen because here lie important emission lines tracing accretion and ejection (e.g. H I and [Fe II]), and also because of the presence of several diagnostic photospheric absorption features that, if detected, are suitable for spectral classification of the forming star.

All spectra were obtained using a $0.3^{\prime \prime}$ slit, providing a final nominal spectral resolution of about 10000 in the $H$ band and 8900 in the $K$ band. The slit was aligned parallel to the jet. All the work on the raw images was performed using the IRAF software package, following the standard procedures for bad pixel removal, flat-fielding, and sky subtraction. Spectra of 
standard stars were acquired at airmasses similar to those of the scientific spectra and used, after removal of any intrinsic line, to correct for the telluric absorptions and to calibrate the flux scale.

Observations of science targets and standards were performed with similar seeing conditions $\left(\sim 0.6^{\prime \prime}\right.$ for HH34 and HH46 IRS and $\sim 1^{\prime \prime}$ for HH26 IRS), so that flux losses are assumed to be comparable in both cases for point-like objects, and no correction was applied to the spectra of the sources (for jets see Sect. 4.2).

From the calibrated spectra we derived an estimate of the magnitudes in the $H$ and $K$ bands, which we list in Table 1, together with the 2MASS magnitudes. The agreement is very good for HH34 IRS, while for the other sources, the differences are $<0.6$ mag except for the $H$ magnitude of HH46 IRS; in this case, however, the 2MASS $H$ magnitude appears to be strangely high, being almost equal to the $K$ magnitude, providing a $J-H$ colour unusual for this kind of sources.

Wavelength calibration was performed using the atmospheric $\mathrm{OH}$ emission lines whenever possible, or, alternately, using Xenon/Argon lamp lines, always refining the alignment against known atmospheric absorption features. The described procedure leads to a calibration error that we estimate as of the order of $0.1 \AA$ for the $H$ band and the first $K$ band segments, and $0.2 \AA$ for the other $K$ band spectral region investigated.

Velocity information on the observed lines ( $V_{\text {LSR }}$ and $\Delta V$ ) was obtained through a Gaussian fit. The uncertainty on the line widths is of about $10 \mathrm{~km} \mathrm{~s}^{-1}$, as we estimate considering the typical difference between the fitted FWHM and the one directly measured on the spectra. Concerning radial velocities, the contribution $V_{\text {cloud }}$ of the star-forming clouds hosting the sources, i.e. $11 \mathrm{~km} \mathrm{~s}^{-1}$ for HH26 IR (Gibb et al. 1995), $8.5 \mathrm{~km} \mathrm{~s}^{-1}$ for HH34 IRS (Anglada et al. 1995), and $8 \mathrm{~km} \mathrm{~s}^{-1}$ for HH46 IRS in Vela (Chernin \& Masson 1991), was subtracted from the measured $V_{\mathrm{LSR}}$.

\section{Results}

\subsection{Observed spectral features}

The continuum-normalised spectra of the three sources are shown in Figs. 1-3. The spectra are characterised by the presence of several emission lines, while there seems to be no evidence of absorption features from the central object photosphere. Depending on the rms noise measured on the spectra, we estimate upper limits on the line/continuum ratio of the undetected photospheric lines between $\sim 5-30 \%$. These ratios indicate the minimum absorption line equivalent width measurable for a given spectrum. Hence, a lower limit to the veiling can be inferred using the relationship $r=E W / E W^{\prime}-1$, connecting the veiling $r$ to the intrinsic $(E W)$ and measured $\left(E W^{\prime}\right)$ equivalent width of a line. Assuming a late $\mathrm{K}$ spectral type for the sources, as the typical intrinsic equivalent widths around $1.5 \AA$ for the strongest absorption features in the $K$ band in this type of stars (e.g. Wallace \& Hinkle 1997), one derives veilings $r_{K} \gtrsim 1,2,5$ for HH46 IRS, HH26 IRS and HH34 IRS, respectively.

Among the emission lines, there are several $\mathrm{H}_{2}$ and [Fe II] transitions coming from the jet, as shown by these lines being not confined to the source but appearing extended in the jet direction in the spectral images. The [Fe II] lines trace regions with moderate ionisation, temperatures between 8000 and $15000 \mathrm{~K}$, and electron densities up to $10^{5} \mathrm{~cm}^{-3}$, which are commonly found in the initial part of a jet, close to the source. Rovibrational $\mathrm{H}_{2}$, on the other hand, is excited in a molecular gas at temperatures of the order of 2000-3000 K and total densities of $\sim 10^{4}-10^{5} \mathrm{~cm}^{-3}$. While HH34 IRS and HH46 IRS show
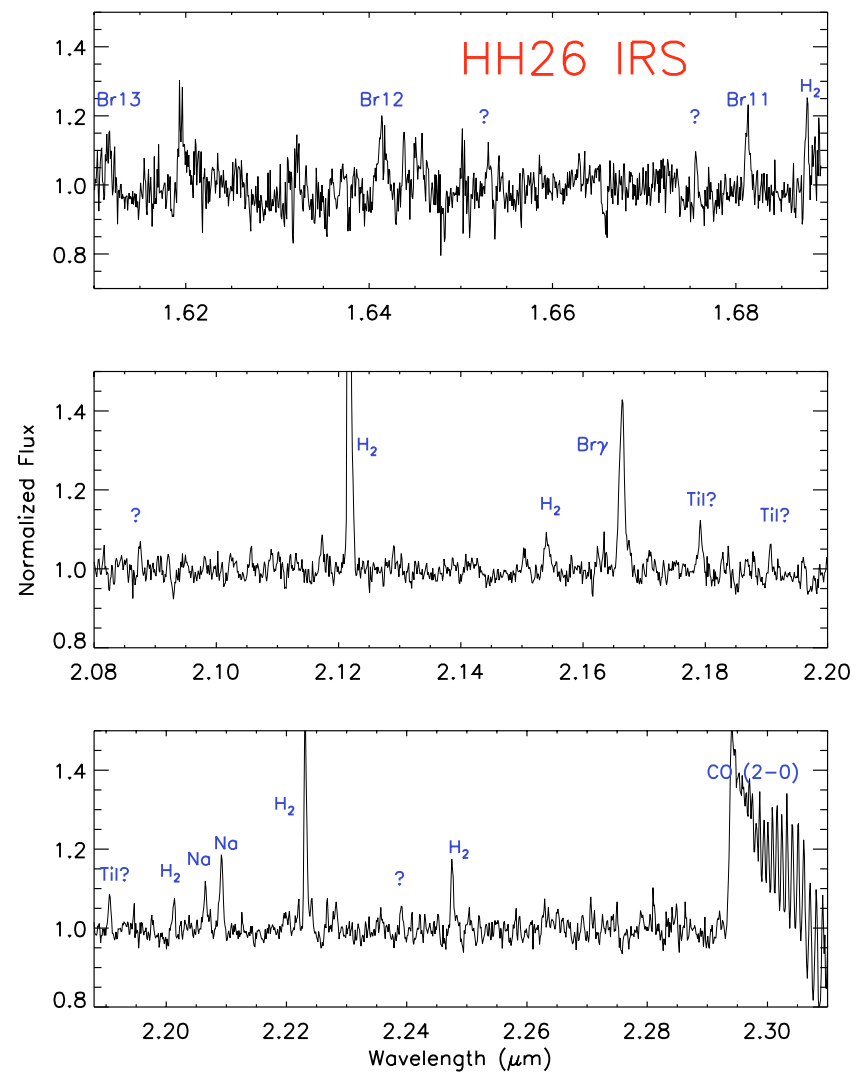

Fig. 1. The $H$ and $K$ band normalised spectra of HH26 IRS.

both [Fe II] and $\mathrm{H}_{2}$ lines, in $\mathrm{HH} 26$ IRS only the latter are detected. This could be an indication that the jet has low excitation conditions or it is composed mostly of molecular material. Similar molecular jets have been found in a small number of Class I sources (Davis et al. 2001). A full account of the jet physical properties derivable from the detected emission lines will be given elsewhere (Garcia Lopez et al. 2007; Calzoletti et al. 2007, in preparation).

Different permitted emission lines are also detected, the most prominent being the $\mathrm{H}$ I recombination lines of the Brackett series (see Fig. 4) and the NaI doublet lines around $2.20 \mu \mathrm{m}$ (Fig. 5), which are observed in HH34 IRS and HH26 IRS. These lines, whose emission is confined toward the source, trace high density gas excited either in the accretion region or at the base of stellar/disc winds. The H I lines have large widths as is usual in these kinds of sources (e.g. Nisini et al. 2005a); the Bry line in HH34 IRS has a width of about $240 \mathrm{~km} \mathrm{~s}^{-1}$, while in the other sources it is about $150 \mathrm{~km} \mathrm{~s}^{-1}$. The peak of the emission is just slightly redshifted, but the profile is not always symmetric, as it extends more into the blue in HH26 IRS. In the case of HH46 IRS, the line profile is difficult to interpret since the line is merged with another prominent emission feature whose identification is not certain, although the wavelength $(2.165 \mu \mathrm{m})$ is compatible with He I. The velocity shift of about $-200 \mathrm{~km} \mathrm{~s}^{-1}$ (similar to the one measured on Fe II) could indicate a $\mathrm{Br} \gamma$ high-velocity component associated with the Fe II jet but confined to the source and not extended along the jet axis. Such a high-velocity component is, however, not detected in the other Brackett series lines. Br $\gamma$ equivalent widths measured on the sources span from -4.8 to $-8.9 \AA$ (see Table 8 ).

Band-head emission from CO is also detected in the sources, even though the emission is much weaker in HH46 IRS. This is 

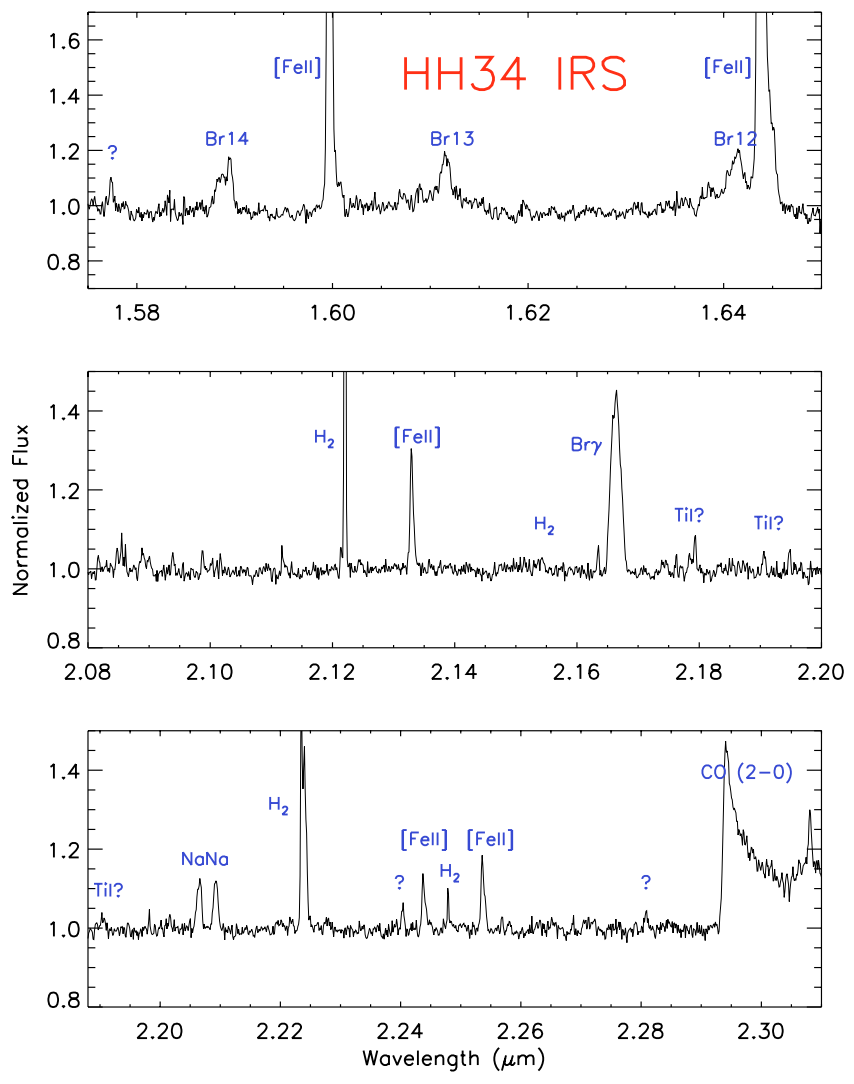

Fig. 2. The $H$ and $K$ band normalised spectra of HH34 IRS.

a tracer for dense molecular gas at $T \sim 2000-3000 \mathrm{~K}$, usually interpreted as a sign of either an active accretion disc or a neutral wind (e.g. Carr 1989; Carr et al. 1993; Najita et al. 1996).

Finally, we detect other lines for which we provide tentative identifications, namely Ti I lines at 2.179 and $2.190 \mu \mathrm{m}$ (visible in HH26 IRS and also in HH34 IRS) and other features that remain unidentified (e.g. at $2.239 \mu \mathrm{m}$ in HH26 IRS and at 2.240, $2.281 \mu \mathrm{m}$ in HH34 IRS). The Ti I lines (that are well-detected especially $\mathrm{HH} 26$ IRS) are broader than $\mathrm{H}_{2}$ lines, while they basically have the same width as the $\mathrm{Na}$ I doublet lines (around $80 \mathrm{~km} \mathrm{~s}^{-1}$ ), thus suggesting that they probably originate in the same region around the central source.

A list of all the observed emission features with an $\mathrm{S} / \mathrm{N}$ greater than 3 and line-widths at least equal to the instrumental resolution is reported in Tables 2-4, together with their fluxes and tentative identifications based on the NIST $^{1}$ and Atomic Line List ${ }^{2}$. Radial velocity information for the brightest lines of the detected series ( $\mathrm{HI} \mathrm{Br} \gamma, \mathrm{H}_{2}$ at $2.12 \mu \mathrm{m}$, Fe II at $1.64 \mu \mathrm{m}$, and $\mathrm{Na}$ I at $2.2 \mu \mathrm{m}$ ) is given in Table 5 .

\subsection{Bolometric luminosity}

The total luminosity $L_{\mathrm{bol}}$ of the sources was derived by integrating the observed spectral energy distributions that include measurements spanning the near IR to the $\mathrm{mm}$ region (see Table 6). The calculation was performed starting from the $J$ band value and considering straight lines (in the $\log (\lambda)-\log \left(\lambda F_{\lambda}\right)$ plan) between available SED points; a final correction at the longest wavelengths was applied assuming that the emission $F_{\lambda}$ is decreasing as $1 / \lambda^{2}$ after the last available observation at $1.3 \mathrm{~mm}$.

\footnotetext{
${ }^{1}$ http://physics.nist.gov/PhysRefData/ASD1

${ }^{2}$ http://www.pa.uky.edu/ peter/atomicdatabases
}
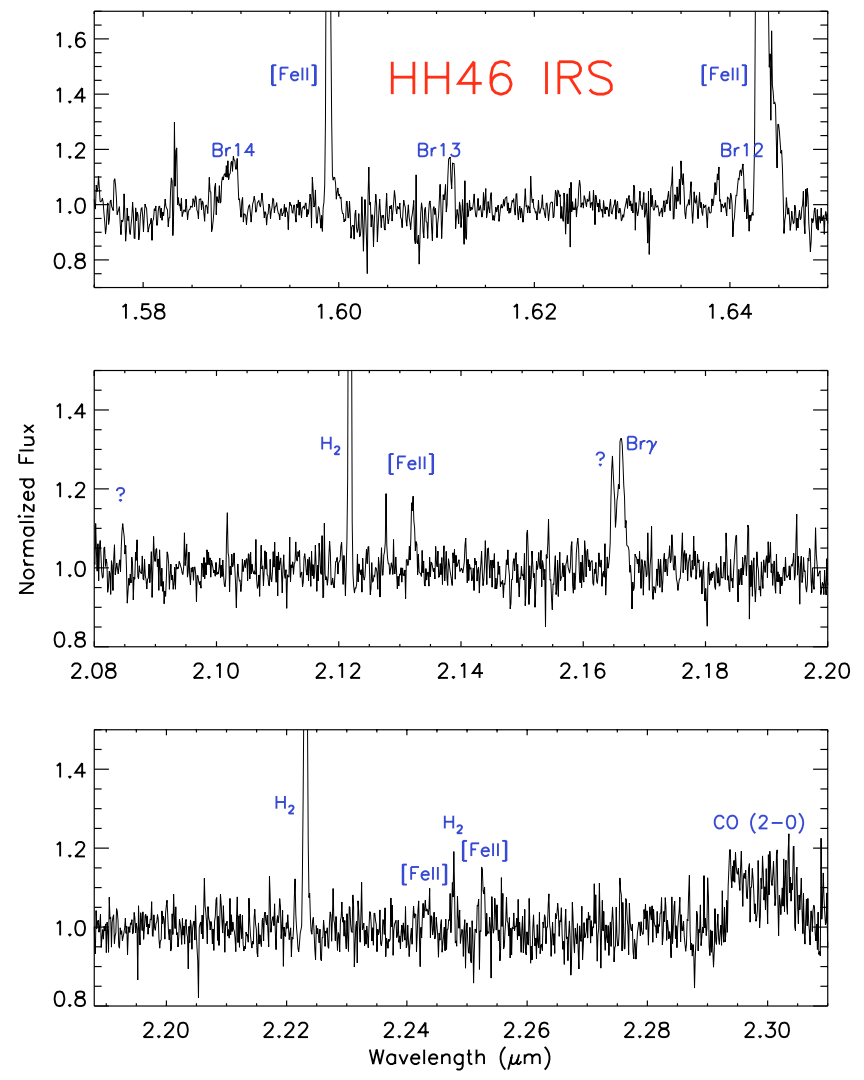

Fig. 3. The $H$ and $K$ band normalised spectra of HH46 IRS.

The derived value might be subject to a bias because of the possible presence of unseen companions contributing to the observed flux or contamination from other stars in the field, especially for the IRAS measurements at 60 and 100 microns. For this reason, we considered recent Spitzer-MIPS images to verify the crowding of the fields at mid IR wavelengths and derive more accurate flux estimates. Results for HH46 IRS at $24 \mu \mathrm{m}$ are given by Noriega-Crespo et al. (2004), while for HH26 IRS and HH34 IRS we used available archive images at 24 and $70 \mu \mathrm{m}$. Spitzer photometry at 24 and 70 microns was considered instead of the respective IRAS fluxes at 25 and 60 microns.

For HH26 IRS, Spitzer photometry shows that contamination from nearby sources is indeed very likely in the IRAS measurements. In fact, the calculated $70 \mu \mathrm{m}$ flux is much lower than the IRAS $60 \mu \mathrm{m}$ point. A luminosity of $9.2 L_{\odot}$ was derived considering the IRAS $100 \mu \mathrm{m}$ point that, however, is likely to be overestimated. Neglecting the 100 point we obtain $4.6 L_{\odot}$. These results are to be regarded as upper and lower limits to the total luminosity of the source.

HH34 IRS does not appear in the IRAS point source catalogue, probably owing to source confusion problems; however, IRAS fluxes were extracted by Cohen \& Schwartz (1987). Actually, the Spitzer images show that strong contamination from other sources is not likely, as the source appears to be quite isolated in the field. On the other hand, the SED displays a very rapid increase at $100 \mu \mathrm{m}$ with respect to the $60 \mu \mathrm{m}$ point. The derived Spitzer fluxes are lower than the IRAS fluxes, although the discrepancy is not as great as in the case of HH26 IRS. The derived total luminosity is about $19.9 L_{\odot}$ considering the IRAS points and $12.4 L_{\odot}$ neglecting the $100 \mu \mathrm{m}$ measurement.

Reipurth et al. (2000) found HH46 IRS to be a binary using HST/NICMOS observations. The binary flux ratio in the band of 

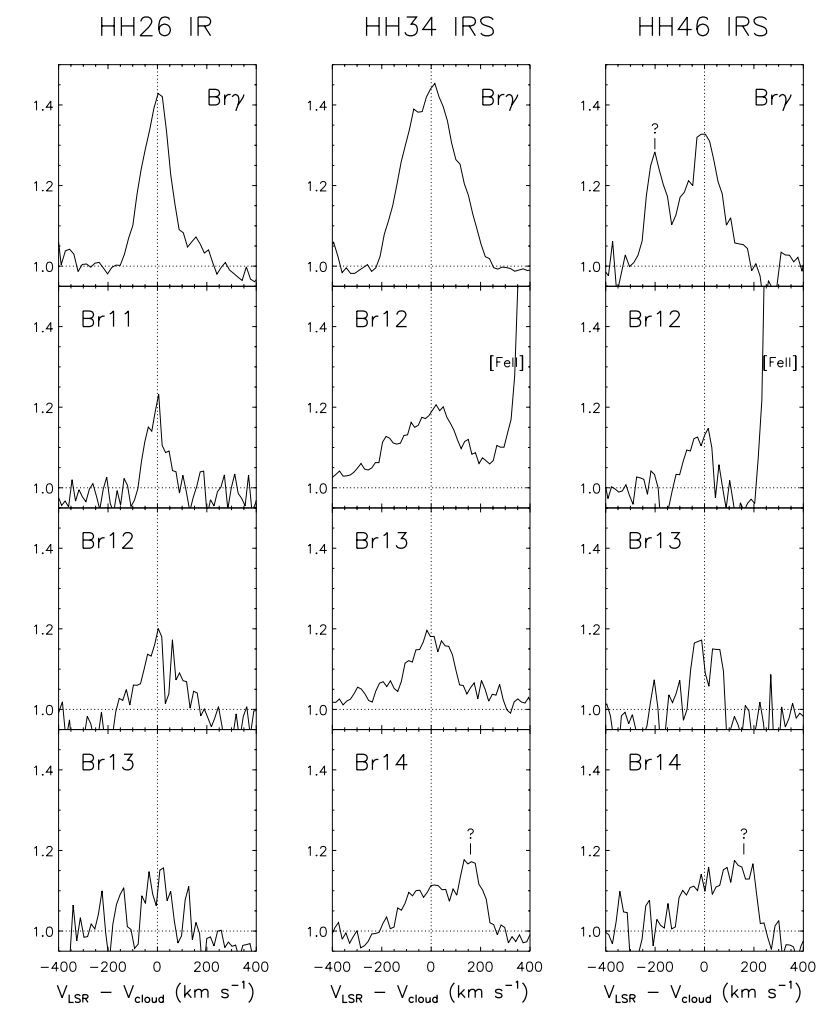

Fig. 4. H I line profiles for (from left to rigth) HH26 IRS, HH34 IRS, and HH46 IRS.
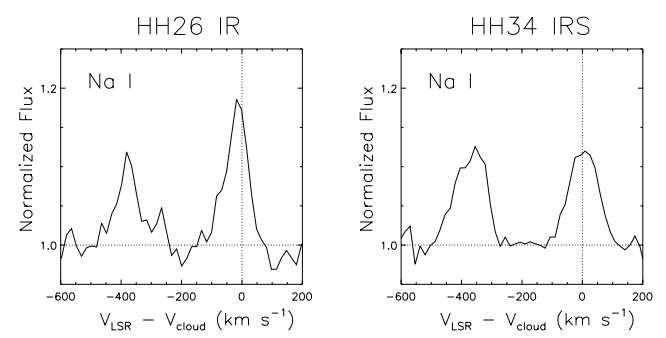

Fig. 5. Na I line profiles for (from left to rigth) HH26 IRS and HH34 IRS.

observation is about 0.7 , but it is impossible to determine which of the two components is responsible for the jet. Also in this case, the Spitzer flux at 24 microns (Noriega-Crespo et al. 2004) is lower than the one measured by IRAS. The inferred total luminosity of the binary is $15.0 L_{\odot}$, which is of course an upper limit to the bolometric luminosity of the HH46 exciting source.

\subsection{Source classification}

The near-IR photometry and the availability of Spitzer data allowed us to refine the classification of the three sources based on their SEDs. The derived $K$-[24] colours are 10.3 mag for HH26 IRS, 12.5 mag for HH34 IRS and 13.3 mag for HH46 IRS. These values indicate that the three sources can indeed be considered as Class I or younger, following Rebull et al. (2007, in press) who classify as Class I sources those having a $K-Q$ colour greater than 8.3. The near-IR $J-H$ and $H-K$ colours of HH34 IRS and HH26 IRS are instead typical of embedded T Tauri stars (Meyer et al. 1997). The HH46 IRS colours are rather peculiar, since the source falls to the left of the reddened main-sequence star locus. As we mention in Sect. 4.1, however, the displacement of the sources in this colour-colour
Table 2. Emission features in HH26 IRS

\begin{tabular}{lccl}
\hline \hline $\begin{array}{l}\lambda \\
(\mu \mathrm{m})\end{array}$ & $\begin{array}{c}F \pm \Delta F \\
\left(10^{-16} \mathrm{erg} \mathrm{s}^{-1} \mathrm{~cm}^{-2}\right)\end{array}$ & $\begin{array}{c}F W H M \\
\left(\mathrm{~km} \mathrm{~s}^{-1}\right)\end{array}$ & $\mathrm{ID}$ \\
\hline 1.6116 & $1.2 \pm 0.7$ & 140 & $\mathrm{Br} \mathrm{13}$ \\
1.6413 & $3.6 \pm 1.6$ & 235 & $\mathrm{Br} 12$ \\
1.6530 & $1.0 \pm 0.3$ & 100 & $?$ \\
1.6756 & $0.7 \pm 0.2$ & 45 & $?$ \\
1.6812 & $2.2 \pm 0.5$ & 100 & $\mathrm{Br} 11$ \\
1.6877 & $1.9 \pm 0.3$ & 60 & $\mathrm{H}_{2} 1-0 \mathrm{~S}(9)$ \\
2.0874 & $2.4 \pm 0.5$ & 70 & $?$ \\
2.1216 & $69.0 \pm 0.4$ & 50 & $\mathrm{H}_{2} 1-0 \mathrm{~S}(1)$ \\
2.1541 & $6.9 \pm 1.2$ & 210 & $\mathrm{H}_{2} 2-1 \mathrm{~S}(2)$ \\
2.1663 & $26.0 \pm 0.8$ & 140 & $\mathrm{Br} \gamma$ \\
2.1791 & $4.6 \pm 0.5$ & 80 & $\mathrm{Ti}$ \\
2.1906 & $3.4 \pm 0.6$ & 80 & $\mathrm{Ti} \mathrm{I}$ \\
2.1905 & $3.1 \pm 0.5$ & 70 & $?$ \\
2.2012 & $2.3 \pm 0.4$ & 60 & $\mathrm{H}_{2} 3-2 \mathrm{~S}(3)$ \\
2.2065 & $4.5 \pm 0.6$ & 80 & $\mathrm{Na} \mathrm{I}^{2}$ \\
2.2092 & $7.8 \pm 0.6$ & 95 & $\mathrm{Na} \mathrm{I}^{\mathrm{I}}$ \\
2.2231 & $15.9 \pm 0.4$ & 60 & $\mathrm{H}_{2} 1-0 \mathrm{~S}(0)$ \\
2.2391 & $2.3 \pm 0.5$ & 65 & $?$ \\
2.2475 & $6.6 \pm 0.4$ & 75 & $\mathrm{H}_{2} 2-1 \mathrm{~S}(1)$ \\
2.2940 & $200.2 \pm 1.5$ & $\ldots$ & $\mathrm{CO}(2-0)^{a}$ \\
\hline
\end{tabular}

Notes. Fluxes are not corrected for extinction. ${ }^{a}$ measured up to $2.305 \mu \mathrm{m}$.

Table 3. Emission features in HH34 IRS.

\begin{tabular}{|c|c|c|c|}
\hline $\begin{array}{l}\lambda \\
(\mu \mathrm{m})\end{array}$ & $\begin{array}{c}F \pm \Delta F \\
\left(10^{-16} \mathrm{erg} \mathrm{s}^{-1} \mathrm{~cm}^{-2}\right)\end{array}$ & $\begin{array}{l}F W H M \\
\left(\mathrm{~km} \mathrm{~s}^{-1}\right)\end{array}$ & ID \\
\hline 1.5773 & $1.5 \pm 0.3$ & 85 & $?$ \\
\hline 1.5888 & $4.2 \pm 0.7$ & 265 & Br14 \\
\hline 1.5895 & $2.3 \pm 0.3$ & 90 & $?$ \\
\hline 1.5996 & $37.6 \pm 0.4$ & 100 & {$[\mathrm{Fe} \mathrm{II}]$} \\
\hline 1.6115 & $4.3 \pm 0.6$ & 180 & Br13 \\
\hline 1.6413 & $7.9 \pm 0.1$ & 290 & Br12 \\
\hline 1.6437 & $156.0 \pm 1.0$ & 100 & {$[\mathrm{Fe} \mathrm{II}]$} \\
\hline 2.1220 & $22.0 \pm 0.1$ & 15 & $\mathrm{H}_{2} 1-0 \mathrm{~S}(1)$ \\
\hline 2.1329 & $8.7 \pm 0.5$ & 80 & {$[\mathrm{Fe}$ II $]$} \\
\hline 2.1545 & $1.1 \pm 0.3$ & 60 & $\mathrm{H}_{2} 2-1 \mathrm{~S}(2)$ \\
\hline 2.1663 & $39.0 \pm 0.1$ & 240 & $\mathrm{Br} \gamma$ \\
\hline 2.1793 & $2.5 \pm 0.5$ & 55 & Ti I \\
\hline 2.1902 & $3.5 \pm 1.1$ & 170 & Ti I? \\
\hline 2.2016 & $1.2 \pm 0.3$ & 45 & $\mathrm{H}_{2} 3-2 \mathrm{~S}(3)$ \\
\hline 2.2065 & $8.3 \pm 0.7$ & 115 & $\mathrm{NaI}$ \\
\hline 2.2092 & $7.9 \pm 0.7$ & 110 & $\mathrm{NaI}$ \\
\hline 2.2234 & $9.1 \pm 0.2$ & 10 & $\mathrm{H}_{2} 1-0 \mathrm{~S}(0)$ \\
\hline 2.2239 & $34.0 \pm 0.7$ & 95 & {$[\mathrm{Fe}$ II $]$} \\
\hline 2.2403 & $2.3 \pm 0.4$ & 55 & $?$ \\
\hline 2.2438 & $6.3 \pm 0.6$ & 85 & {$[\mathrm{Fe}$ II $]$} \\
\hline 2.2478 & $2.2 \pm 0.2$ & 10 & $\mathrm{H}_{2} 2-1 \mathrm{~S}(1)$ \\
\hline 2.2536 & $8.8 \pm 0.5$ & 85 & {$[\mathrm{Fe}$ II $]$} \\
\hline 2.2807 & $2.1 \pm 0.6$ & 75 & $?$ \\
\hline 2.2940 & $184.0 \pm 1.5$ & $\ldots$ & $\mathrm{CO}(2-0)^{a}$ \\
\hline
\end{tabular}

Notes. Fluxes are not corrected for extinction. ${ }^{a}$ measured up to $2.305 \mu \mathrm{m}$.

diagram is not indicative of the true amount of extinction, since the large quantity of scattered light makes the star appear bluer and less reddened (see e.g. Massi et al. 1999; Stark et al. 2006). Contamination due to light scattered by the circumstellar disc is indeed very likely in all our sources, which have estimated disc inclination angles, with respect to the line of sight, of $20-30^{\circ}$.

That the sources are in an early stage of evolution is also indicated by their relatively massive dust envelopes $\left(0.2-1 M_{\odot}\right.$ Reipurth et al. 1993; Lis et al. 1999). Indeed, the ratio between 
Table 4. Emission features in HH46 IRS.

\begin{tabular}{lccl}
\hline \hline $\begin{array}{l}\lambda \\
(\mu \mathrm{m})\end{array}$ & $\begin{array}{c}F \pm \Delta F \\
\left(10^{-16} \mathrm{erg} \mathrm{s}^{-1} \mathrm{~cm}^{-2}\right)\end{array}$ & $\begin{array}{c}F W H M \\
\left(\mathrm{~km} \mathrm{~s}^{-1}\right)\end{array}$ & ID \\
\hline 1.5889 & $5.1 \pm 0.8$ & 310 & $\mathrm{Br} \mathrm{14}$ \\
1.5989 & $18.3 \pm 0.3$ & 60 & {$[\mathrm{Fe} \mathrm{II}]$} \\
1.6114 & $2.7 \pm 0.4$ & 150 & $\mathrm{Br} \mathrm{13}$ \\
1.6387 & $12.1 \pm 0.5$ & 100 & $?$ \\
1.6411 & $21.1 \pm 0.5$ & 120 & $\mathrm{Br} \mathrm{12}$ \\
1.6429 & $173.2 \pm 0.4$ & 65 & {$[\mathrm{Fe} \mathrm{II]}$} \\
2.0847 & $1.3 \pm 0.4$ & 80 & $?$ \\
2.1218 & $14.8 \pm 0.3$ & 45 & $\mathrm{H}_{2} 1-0 \mathrm{~S}(1)$ \\
2.1321 & $2.4 \pm 0.4$ & 110 & {$[\mathrm{Fe}$ II] } \\
2.1647 & $3.0 \pm 0.3$ & 80 & $?$ \\
2.1662 & $7.4 \pm 0.6$ & 175 & $\mathrm{Br} \gamma$ \\
2.2231 & $20.3 \pm 0.7$ & 80 & $\mathrm{H}_{2} 1-0 \mathrm{~S}(0)$ \\
2.2432 & $5.1 \pm 2.0$ & 350 & {$[\mathrm{Fe} \mathrm{II}]$} \\
2.2477 & $2.5 \pm 0.5$ & 50 & $\mathrm{H}_{2} 2-1 \mathrm{~S}(1)$ \\
2.2526 & $3.4 \pm 0.9$ & 85 & {$[\mathrm{Fe}$ II] } \\
2.2936 & $44.8 \pm 0.9$ & $\ldots$ & $\mathrm{CO}(2-0)^{a}$ \\
\hline
\end{tabular}

Notes. Fluxes are not corrected for extinction. ${ }^{a}$ measured up to $2.305 \mu \mathrm{m}$.

Table 5. Radial velocities $\left(V_{\mathrm{LSR}}-V_{\text {cloud }}\right)$ measured on the brightest lines of the detected series.

\begin{tabular}{lcccc}
\hline \hline Source & $\begin{array}{c}\mathrm{HI} \mathrm{Br} \gamma \\
\left(\mathrm{km} \mathrm{s}^{-1}\right)\end{array}$ & $\begin{array}{c}\text { Fe II }(1.64 \mu \mathrm{m}) \\
\left(\mathrm{km} \mathrm{s}^{-1}\right)\end{array}$ & $\begin{array}{c}\mathrm{H}_{2}(2.12 \mu \mathrm{m}) \\
\left(\mathrm{km} \mathrm{s}^{-1}\right)\end{array}$ & $\begin{array}{c}\mathrm{Na} \mathrm{I} \\
\left(\mathrm{km} \mathrm{s}^{-1}\right)\end{array}$ \\
\hline HH 26 IRS & -3 & $\ldots$ & -56 & -10 \\
HH 34 IRS & -3 & $-88^{a}$ & -11 & 3 \\
HH 46 IRS & -3 & -200 & -11 & $\ldots$ \\
\hline
\end{tabular}

Notes. We estimate an uncertainty of $5 \mathrm{~km} \mathrm{~s}^{-1}$ for the values reported here. ${ }^{a}$ the line is actually double-peaked, with a blue peak $V_{\mathrm{LSR}}-$ $V_{\text {cloud }}=-93.5 \mathrm{~km} \mathrm{~s}^{-1}$ and a red peak $V_{\mathrm{LSR}}-V_{\text {cloud }}=-62.5 \mathrm{~km} \mathrm{~s}^{-1}$.

$L_{\text {bol }}$ and the sub-mm luminosity measured longward of $350 \mu \mathrm{m}$ $\left(L_{\text {submm }}\right)$, estimated from the photometric data, is rather low in all the sources ( $\sim 60$ for HH26 IRS, $\sim 70$ for HH34 IRS and $\sim 160$ for HH46 IRS), which would indicate that they actually satisfy the Class 0 criterium $L_{\text {bol }} / L_{\text {submm }}<200$ as defined by André et al. (1993). Moreover, comparing the observed SEDs with the grid of YSO SED models by Whitney et al. (2003) and Robitaille et al. (2006), we found that models for evolved Class 0 sources provide better fits to the observational data than those for Class I/IIs, although some parameters, such as the depth of the silicate feature, deviate a lot with respect to the observed values. In all cases, these findings strongly suggest that the sources are indeed very young and are not more evolved (Class II) objects.

\section{Analysis and physical parameters}

\subsection{Extinction, accretion, and stellar parameters}

The fraction of the total luminosity due to accretion $\left(L_{\text {acc }}\right)$ must be determined in order to estimate the mass accretion rate. Nisini et al. (2005a) derived this quantity for a sample of young sources, directly determining the spectral type and veiling from the analysis of the absorption features from the stellar photosphere, then inferring the stellar parameters and luminosity $L_{*}$, from which the accretion luminosity can be obtained $\left(L_{\mathrm{acc}}=\right.$ $\left.L_{\text {bol }}-L_{*}\right)$. However, our spectra show no evidence of welldetected $(S / N \gtrsim 3)$ absorption features from the photosphere, indicating the presence of a strong veiling, so that an alternative method has to be used to derive the accretion luminosity.
Table 6. Photometric measurements available in the literature.

\begin{tabular}{llll}
\hline \hline Measurements & HH26 IRS & HH34 IRS & HH46 IRS \\
\hline 2MASS J (mag) & 16.77 & 15.06 & 14.20 \\
2MASS H (mag) & 14.07 & 13.60 & 12.88 \\
2MASS K (mag) & 11.88 & 12.38 & 12.72 \\
\hline IRAS 12 $\mu \mathrm{m}(\mathrm{Jy})$ & 1.93 & 0.65 & 0.81 \\
IRAS $25 \mu \mathrm{m}(\mathrm{Jy})$ & 5.13 & 8.49 & 6.31 \\
IRAS $60 \mu \mathrm{m}(\mathrm{Jy})$ & 20.87 & 31.7 & 26.31 \\
IRAS $100 \mu \mathrm{m}(\mathrm{Jy})$ & 67.93 & 94.8 & 58.27 \\
\hline MSX $8.28 \mu \mathrm{m}(\mathrm{Jy})$ & $\ldots$ & 0.19 & $\ldots$ \\
MSX 14.65 $\mu \mathrm{m}(\mathrm{Jy})$ & $\ldots$ & 1.54 & $\ldots$ \\
MSX 21.30 $\mu \mathrm{m}(\mathrm{Jy})$ & $\ldots$ & 5.54 & $\ldots$ \\
\hline SPITZER $24 \mu \mathrm{m}(\mathrm{Jy})$ & $1.8^{(8)}$ & $6.7^{(8)}$ & $3.9^{(7)}$ \\
SPITZER 70 $\mu \mathrm{m}(\mathrm{Jy})$ & $7.9^{(8)}$ & $23.3^{(8)}$ & $\ldots$ \\
\hline $350 \mu \mathrm{m}(\mathrm{Jy})$ & $6.30^{(3)}$ & $\ldots$ & $\ldots$ \\
$800 \mu \mathrm{m}(\mathrm{Jy})$ & $\ldots$ & $\ldots$ & $1.12^{(4)}$ \\
$850 \mu \mathrm{m}(\mathrm{Jy})$ & $1.19^{(2)}$ & $3.4^{(1)}$ & $\ldots$ \\
$1.1 \mathrm{~mm}(\mathrm{Jy})$ & $\ldots$ & $\ldots$ & $0.34^{(4)}$ \\
$1.3 \mathrm{~mm}(\mathrm{Jy})$ & $0.32^{(3)}$ & $0.42^{(6)}$ & $0.18^{(6)}$ \\
$3.6 \mathrm{~cm}(\mathrm{mJy})$ & $\ldots$ & $0.16^{(5)}$ & $\ldots$ \\
\hline
\end{tabular}

References. (1) Johnstone \& Bally (2006); (2) Johnstone et al. (2001); (3) Lis et al. (1999); (4) Correia et al. (1998); (5) Rodríguez \& Reipurth (1996); (6) Reipurth et al. (1993); (7) Noriega-Crespo et al. (2004); (8) Spitzer archive.
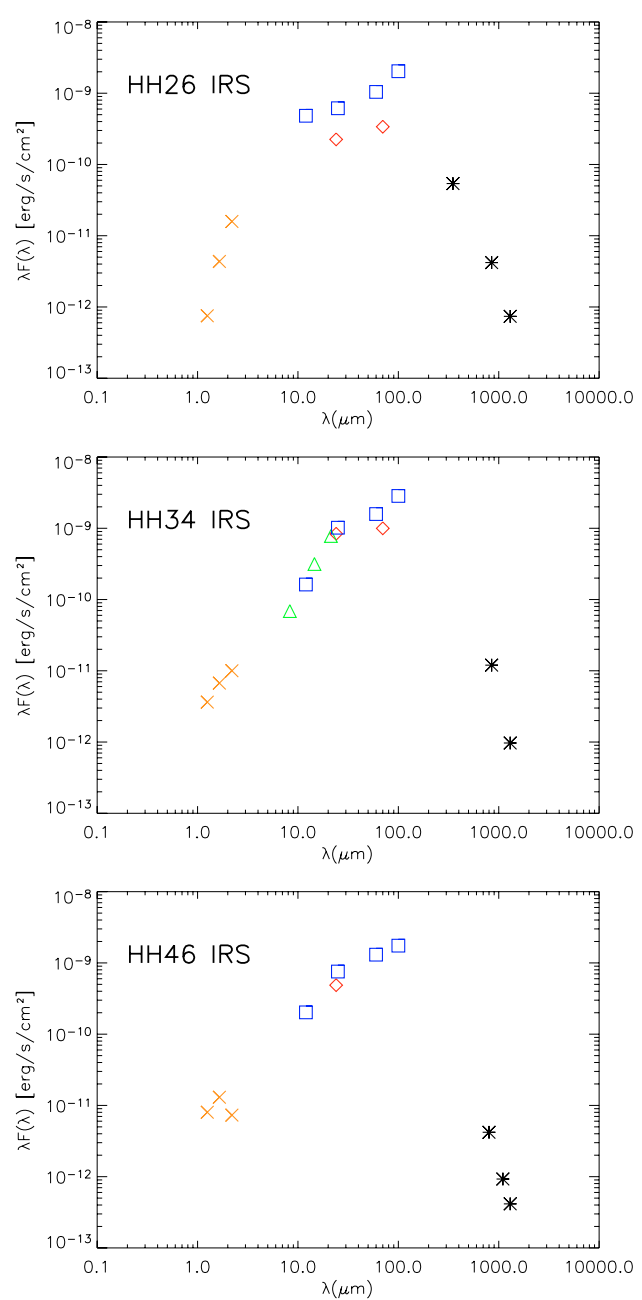

Fig. 6. Spectral energy distributions of the three sources, derived from the values reported in Table 6. Points from different instruments are displayed with different symbols (2MASS: crosses, MSX: triangles, IRAS: squares, SPITZER: diamonds, far IR/mm measurements: stars. 
We have therefore considered the relationship found by Muzerolle et al. (1998a) on the basis of observing T Tauri stars, in which $L_{\text {acc }}$ is directly related to the (extinction-corrected) $\mathrm{H}$ I Br $\gamma$ flux:

$\log \frac{L_{\mathrm{acc}}}{L_{\odot}}=(1.26) \log \frac{L_{\mathrm{Br} \gamma}}{L_{\odot}}+(4.43)$.

Such an approach implies considering this relation is still valid for the more embedded sources of our sample; the only observational evidence of this assumption has been given so far for three sources in the R CrA star-forming region (Nisini et al. 2005a).

In order to retrieve absolute $\mathrm{Br} \gamma$ luminosities from observed fluxes, it is necessary to correct them for the extinction and thus to measure the value of $A_{\mathrm{K}}$ toward the sources. Estimates of the extinction can be inferred by adopting standard methods based on the analysis of the spectral features. For HH46 IRS, considering the silicate absorption feauture at $9.7 \mu \mathrm{m}$ observed by Spitzer (Boogert et al. 2004) and using the relation by Mathis (1998), we obtain an $A_{V}$ value between 32 and $38 \mathrm{mag}$, corresponding to an $A_{K}$ 3.5-4.2 adopting the Rieke \& Lebofsky (1985) extinction law. In the case of HH26 IRS, measurements of the ice absorption feature at $3 \mu \mathrm{m}$ (Simon et al. 2004) and the relationship by Tanaka et al. (1990) provide an $A_{V}$ value of the order of 29 mag $\left(A_{K} \sim 3.2\right)$. We note, however, that the ISM relationships between dust features and extinction, derived for the ISM, may not be strictly valid for dust in the circumstellar environment of YSOs (e.g. Chiar et al. 2007).

Finally, for HH34 IRS only a lower limit of $A_{\mathrm{V}}=7$ mag can be taken by adopting the value recently estimated by Podio et al. (2006) from the ratio of [Fe II] lines excited in the jet region at a distance less than $\sim 1^{\prime \prime}$ from the source.

Given these problems, since reliably determining the extinction is crucial for deriving the accretion luminosity from $\operatorname{Br} \gamma$, we tried to use a different and independent method of computing "self-consistent" $A_{K}$, based on the observed magnitudes and on reasonable assumptions for the stellar properties of the objects. Indeed, the intrinsic stellar luminosity is given by

$\log \left(L_{*} / L_{\odot}\right)=-0.4\left(M_{\mathrm{bol}}-M_{\mathrm{bol}, \odot}\right)$

where

$M_{\mathrm{bol}}=B C+M_{K}+(V-K)_{*}$.

The absolute magnitude $M_{K}$ in Eq. (3) depends on the observed magnitude $m_{K}$, the extinction $A_{K}$, the veiling $r_{K}$, and the distance modulus DM,

$M_{K}=m_{K}+2.5 \log \left(1+r_{K}\right)-A_{K}-D M$,

while the values of the bolometric correction $B C$ and the intrinsic stellar colour $(V-K)_{*}$ depend on the spectral type and age of the objects. Thus, if we assume that our sources are late-type stars located on the birthline and use the parameters provided by a set of evolutionary models, we can obtain an estimate of $A_{K}$ from the observed $K$-band magnitude and veiling of the objects.

On this basis, we adopted a procedure that allowed us to derive the different physical quantities in a self-consistent way. Namely, we varied the $A_{K}$ until we have found a value able to reconcile the stellar luminosity given by Eq. (2) with the value given by $L_{\mathrm{bol}}-L_{\mathrm{acc}}$, where $L_{\mathrm{acc}}$ is derived from the extinction-corrected (by the considered $A_{K}$ ) $\mathrm{Br} \gamma$ line emission using Eq. (1).

For example, we can easily derive an extinction value $A_{V}=$ 29 mag for HH26 IRS (such as the one derived from the ice feature) that would lead to a stellar luminosity that is smaller than the value given by $L_{\mathrm{bol}}-L_{\mathrm{acc}}\left(\Delta L_{*} \sim 2.1 L_{\odot}\right)$, pointing to an inconsistency in the independently inferred $A_{K}$ and $L_{*}$ values.

We carried out the described procedure assuming the birthline location of Palla \& Stahler (1993) and considering the colours and bolometric correction given by Siess et al. (2000) to convert $L_{*}$ into $M_{K}$ (Eqs. (2) and (3)); thus, we found the extinction providing "consistent" $L_{*}$ values. We derived for HH26 IRS an $A_{K}=4.1 \mathrm{mag}$ with a spectral type $\mathrm{K} 7\left(L_{*}=3.7 L_{\odot}, R_{*}=\right.$ $4.1 R_{\odot}, M_{*}=0.6 M_{\odot}$ ) and for HH34 IRS an $A_{K}=4.9$ mag with a spectral type $\mathrm{M} 0\left(L_{*}=2.9 L_{\odot}, R_{*}=3.9 R_{\odot}, M_{*}=0.5 M_{\odot}\right)$.

In the case of HH46 IRS we know that the source is a binary, but the contribution from the companion remains unknown. Assuming that the bolometric luminosity is equally distributed between the two sources, the best results are obtained considering a K5 star with $A_{K}=4.9 \mathrm{mag}\left(L_{*}=6.0 L_{\odot}, R_{*}=4.2 R_{\odot}\right.$, $\left.M_{*}=1.2 M_{\odot}\right)$. If we assume that the contribution to the bolometric luminosity of our considered target is higher than the companion, we find that it is not possible to obtain consistent results for $L_{*}$ lower than $10 L_{\odot}$; exploring the possibility of a more massive star, we can still find consistency with an $A_{K} \sim 5.3 \mathrm{mag}$ and a spectral type $\mathrm{G} 9 / \mathrm{K} 0\left(L_{*}=12.5 L_{\odot}, R_{*}=4.3 R_{\odot}\right.$, $\left.M_{*}=2.8 M_{\odot}\right)$. However, in this case the bolometric contribution of the companion (considering a derived $L_{\text {acc }} \sim 2.4 L_{\odot}$ ) would basically be zero, which appears unlikely. In Table 7 we report the parameters derived for the three sources adopting the analysis just described.

Providing self-consistent values of the parameters, we are confident that these are better determinations of the extinction compared to the previous ones, also considering the already mentioned problems that may arise using the relationships established for the ISM for dust around YSOs. Moreover, adopting the extinction values from spectral features and jet-line ratios, we would infer accretion luminosities $\sim 1 L_{\odot}$ for HH26 IRS, $\sim 0.1 L_{\odot}$ for HH34 IRS and $0.3-0.7 L_{\odot}$ for HH46 IRS, which represent only a small fraction of the total luminosity of the sources ( 1-20\%), thus implying that most of the source luminosity should be contributed by the stellar photosphere. However, this contrasts with the early evolutionary state of the objects and with the high $K$-band veiling values we find.

Conversely, the accretion luminosities that we infer when adopting our "self-consistent" extinction values are $3.2 L_{\odot}$ for HH26 IRS, $13.3 L_{\odot}$ for HH34 IRS, and $1.5 L_{\odot}$ for HH46 IRS. The estimated accretion to total luminosity ratios are therefore $\sim 0.5$ for HH26 IRS, $\sim 0.8$ for HH34 IRS, and $\sim 0.2$ for HH46 IRS (see Table 7), where we considered a mean value of the $L_{\text {bol }}$ ranges given in Table 1 for HH26 IRS and HH34 IRS.

From these accretion luminosities we then derived an estimate of the mass accretion rate $\dot{M}_{\text {acc }}$, using the formula for disc accretion (Gullbring et al. 1998):

$\dot{M}_{\mathrm{acc}}=\frac{L_{\mathrm{acc}} R_{*}}{G M_{*}}\left(1-\frac{R_{*}}{R_{\mathrm{i}}}\right)^{-1}$

where $R_{*}$ and $M_{*}$ are the stellar radius and mass, respectively, and $R_{\mathrm{i}}$ is the inner radius of the accretion disc. We consider the masses and radii derived from Siess models during the computation of the extinction described above and assume a value of $R_{\mathrm{i}}=5 R_{*}$ for the disc inner radius (e.g. Gullbring et al. 1998). The inferred mass accretion rates are listed in Table 7 and are approximately $10^{-7}$ (HH26 IRS and HH46 IRS) and $10^{-6}$ (HH34 IRS) $M_{\odot} \mathrm{yr}^{-1}$.

All previous calculations were carried out assuming the measured lower limits on veiling. Considering higher $r_{K}$ values 
and performing the computations again leads to increased $A_{K}$ and $L_{\mathrm{acc}}$, lower $L_{*}$ and $M_{*}$, and consequently to greater $L_{\mathrm{acc}} / L_{\mathrm{bol}}$ and $\dot{M}_{\text {acc }}$. In particular, in the case of HH26 IRS, taking a veiling $r_{K}=4$ into account we get a higher $A_{K} \sim 4.4$ with a spectral type M1 and $L_{\text {acc }} \sim 4.1 L_{\odot}$; we would thus obtain $L_{\text {acc }} / L_{\text {bol }} \sim 0.6$ and $\dot{M}_{\text {acc }} \sim 1.6 \times 10^{-6} M_{\odot} \mathrm{yr}^{-1}$.

\subsection{Jet mass flux}

The jet mass flux can be derived from emission-line measurements, providing that the jet is resolved and its velocity known (see e.g. Hartigan et al. 1995; Nisini et al. 2005b). For our purpose of directly comparing the mass loss with the mass accretion rate, we need to determine the mass flux relative to jet component as close as possible to the central source. Jet knots far from the protostars may indeed be related to older episodes of mass ejection, which could be associated to a different mass flux.

For HH46, we estimated the mass flux from the luminosity of the [FeII] $1.64 \mu \mathrm{m}$ line, measured on the first 3 arcsec of the jet length. There are no measurements of the jet diameter in this inner jet section, which is not optically visible: at a distance of $\sim 5$ arcsec, a jet diameter of $\sim 1$ arcsec has been measured by Eislöffel \& Mundt (1994). Since jet diameters increase with distance from the driving source (e.g. Dougados et al. 2000), the diameter in the portion of the jet we are considering should be $\sim 0.6$ arcsec. Thus, in addition to the flux losses due to the seeing, we have also taken a correction factor of 2 into account to retrieve an estimate of the total flux in the considered jet section. We therefore have

$\dot{M}_{\mathrm{loss}}=\mu m_{\mathrm{H}} \times\left(n_{\mathrm{H}} V\right) \times v_{\mathrm{t}} / l_{\mathrm{t}}$

and

$n_{\mathrm{H}} V=L_{1.64 \mu \mathrm{m}}\left(h v A_{i} f_{i} \frac{[\mathrm{Fe}]}{[\mathrm{H}]}\right)^{-1}$,

where $A_{i}, f_{i}$ are the radiative rate and fractional population of the upper level of the considered transition, $[\mathrm{Fe}] /[\mathrm{H}]$ is the total $\mathrm{Fe}$ abundance with respect to hydrogen (assumed $2.82 \times 10^{-5}$, Asplund 2005) and $v_{\mathrm{t}}$ and $l_{\mathrm{t}}$ are the velocity and length of the knot, projected perpendicularly to the line of sight. We assume here that all $\mathrm{Fe}$ is ionised, as is expected in the excitation conditions of $\mathrm{HH}$ objects (Nisini et al. 2002). The fractional population was computed adopting an NLTE statistical equilibrium code (Nisini et al. 2002) with an electron density $n_{\mathrm{e}}=3.7 \times 10^{3} \mathrm{~cm}^{-3}$, estimated from the $[\mathrm{Fe} \mathrm{II}] 1.64 / 1.60 \mathrm{ob}-$ served line ratio. The electron temperature was assumed to be $10^{4} \mathrm{~K}$. The tangential velocity was inferred through the proper motion study of Eislöffel \& Mundt (1994), and it is equal to $170 \mathrm{~km} \mathrm{~s}^{-1}$. The $1.64 \mu \mathrm{m}$ line luminosity was calculated from the observed flux, which was dereddened assuming that the extinction in the considered jet knot is $A_{V}=6.6 \mathrm{mag}$. This value was estimated from the [Fe II] 1.64/1.25 $\mu \mathrm{m}$ line ratio measured by Fernandes (2000), adopting the expected theoretical ratio of Quinet et al. (1996). The resulting mass flux value is about $3 \times 10^{-8} M_{\odot} \mathrm{yr}^{-1}$. This value is about a factor of 3 and 10 lower than the values estimated by Bacciotti \& Eislöffel (1999) and by Hartigan et al. (1994), respectively, through the measurement of the total gas density, and thus the total mass in the jet, following the procedure known as the BE technique (from Bacciotti \& Eislöffel 1999) or adopting a shock model. It has been shown that the mass flux thus inferred may be overestimated due to the assumption that the considered jet area is filled with gas at the derived density (Hartigan et al. 1994; Nisini et al. 2005a). If the shock is caused by jet time-variability, the lack of correction for shock compression will overestimate the time-averaged $\dot{M}_{\text {loss }}$ by a factor of about 5 (Hartigan et al. 1994). On the other hand, Hartigan et al. (1994) also compute a mass flux value of $4.2 \times 10^{-7} M_{\odot} \mathrm{yr}^{-1}$ from the luminosity of the [O I] $6300 \AA$ line, adopting a relationship similar to the one in Eq. (6), although on a jet region much more distant $\left(\sim 10-20^{\prime \prime}\right.$ from the source) than the one we consider.

Part of the above discrepancies may arise from our assumption of solar abundance of gas-phase Fe. This assumption may not be valid if part of iron is still locked in grains along the jet. This is indeed what was found in other jets (HH1 and HH34) by Nisini et al. (2005b) and Podio et al. (2006), who showed that in the inner jet regions only a fraction between 30 and $70 \%$ of all iron may be in gaseous form. If the same also applies to the HH46 jet, then our data give a mass flux up to $\sim 2 \times 10^{-7} M_{\odot} \mathrm{yr}^{-1}$.

The mass flux in the HH34 inner jet has been measured by several authors. The most recent determinations were given by Davis et al. (2003), who used the luminosity of the [Fe II]1.64 $\mu \mathrm{m}$ line, by Podio et al. (2006), who compared different techniques employing both optical and IR lines, and finally by Garcia Lopez et al. (2007), who used the [Fe II]1.64 $\mu \mathrm{m}$ luminosity from the same set of data we are analysing here. All these determinations agree on a value in the range $4-7 \times 10^{-8} M_{\odot} \mathrm{yr}^{-1}$. If we correct the determinations based on [Fe II] line luminosity by the $30 \%$ of iron dust depletion found in the inner jet knot by Podio et al. (2006), we get a mass flux up to $\sim 1.2 \times 10^{-7} M_{\odot} \mathrm{yr}^{-1}$, which also agrees with the determinations by Hartigan et al. (1994).

The jet in $\mathrm{HH} 26$ is seen close to the star only in $\mathrm{H}_{2}$, indicating that the gas is mainly molecular and that the atomic component does not give significantly contributes to the transport of material outwards. We therefore measured the mass flux from the luminosity of the observed $2.12 \mu \mathrm{m} \mathrm{H}_{2}$ line. More explicitly, Eq. (6) can be written here as

$\dot{M}_{\mathrm{loss}}=2 \mu m_{\mathrm{H}} \times\left(N\left(\mathrm{H}_{2}\right) A\right) \times v_{\mathrm{t}} / l_{\mathrm{t}}$

where $N\left(\mathrm{H}_{2}\right)$ is the column density of molecular hydrogen and $A$ the considered emission area. Since $\mathrm{H}_{2}$ is optically thin and quickly thermalised, the total column density can be directly measured from the dereddened line luminosity:

$N\left(\mathrm{H}_{2}\right) A=L_{2.12 \mu \mathrm{m}}\left(h v A_{i} f_{i}\right)^{-1}$

with $f_{i}$ given by the LTE Boltzman population at the gas kinetic temperature. A temperature of $\sim 2000 \mathrm{~K}$ can be estimated by the ratio of the various observed lines. We only considered the first knot of the HH26 microjet, which extends within about 2 arcsec from the central source. As in the case of HH46, we applied a correction of a factor of 3 to take the width of the jet at the knot position into account, which is $\sim 1^{\prime \prime}$ (Chrysostomou et al. 2007). The adopted line flux results consistent with the one measured by Giannini et al. (2004) with a slit of $1^{\prime \prime}$. The tangential velocity was estimated to be around $130 \mathrm{~km} \mathrm{~s}^{-1}$ from the measured radial velocity of $-60 \mathrm{~km} \mathrm{~s}^{-1}$, assuming an inclination angle of $65^{\circ}$ with respect to the line of sight (Davis et al. 1997). Here, a critical parameter is the reddening towards the jet. Giannini et al. (2004) measured an $A_{\mathrm{V}}=2$ on the external HH26A knot; it is, however, likely that the extinction has a sharp increase close to the object, since a value of $A_{V} \sim 38$ has been estimated onsource. A method of estimating $A_{V}$ in the $\mathrm{H}_{2}$ emitting region is to construct a Boltzman diagram with the $\mathrm{H}_{2}$ lines detected on source (Table 3 ) and take the $A_{V}$ value that gives the better alignment of the different transitions on a straight line (see 
Table 7. Derived parameters for the observed targets.

\begin{tabular}{|c|c|c|c|c|c|c|c|c|c|}
\hline Source & $\begin{array}{c}A_{K} \\
(\mathrm{mag})\end{array}$ & $\begin{array}{l}L_{\text {acc }} \\
\left(L_{\odot}\right)\end{array}$ & $\begin{array}{c}L_{*} \\
\left(L_{\odot}\right)\end{array}$ & $\begin{array}{c}M_{*} \\
\left(M_{\odot}\right)\end{array}$ & ST & $L_{\mathrm{acc}} / L_{\mathrm{bol}}$ & $\begin{array}{c}\dot{M}_{\mathrm{acc}} \\
\left(10^{-7} M_{\odot} \mathrm{yr}^{-1}\right)\end{array}$ & $\begin{array}{c}\dot{M}_{\mathrm{loss}} \\
\left(10^{-7} M_{\odot} \mathrm{yr}^{-1}\right)\end{array}$ & $\dot{M}_{\text {loss }} / \dot{M}_{\text {acc }}$ \\
\hline HH26 IRS & $\sim 4.1$ & 3.2 & 3.7 & 0.6 & K7 & $\sim 0.5$ & 8.5 & $0.2-0.5$ & $0.02-0.06$ \\
\hline HH34 IRS & $\sim 4.9$ & 13.3 & 2.9 & 0.5 & M0 & $\sim 0.8$ & 41.1 & $0.4-1.2^{a}$ & $0.01-0.03$ \\
\hline HH46 IRS & $\sim 4.9$ & 1.5 & 6.0 & 1.2 & K5 & $\sim 0.2$ & 2.2 & $0.3-2.0^{b}$ & $0.14-0.90$ \\
\hline HH100-IR & $3.3 \pm 0.3$ & $12 \pm 2$ & $3.1 \pm 0.9$ & $0.3-0.75$ & K5-M0 & 0.80 & $10-20$ & $\cdots$ & \\
\hline IRS2 & $2.4 \pm 0.3$ & $7.7 \pm 2.5$ & $4.3 \pm 1.5$ & $1.1-1.8$ & K2 & 0.60 & $2-3$ & $\ldots$ & $\ldots$ \\
\hline IRS5a & $5.0 \pm 0.6$ & $\sim 0.4$ & $1.6 \pm 0.5$ & $0.4-0.9$ & K5-K7 & 0.15 & $0.2-0.3$ & $\ldots$ & $\ldots$ \\
\hline
\end{tabular}

Notes. Columns show in order: $K$-band extinction, accretion luminosity, stellar luminosity (assuming the star on the birthline), estimated stellar mass and spectral type (using Siess et al. 2000, models), accretion to bolometric luminosity ratio, mass accretion rate, (one-sided) mass loss rate, mass loss rate-to-mass accretion rate ratio. The same results for three Class I sources of the R CrA region (Nisini et al. 2005a) are reported for comparison (see text for details). ${ }^{a}$ Lower value from optical line determinations (Podio et al. 2006); upper value from [Fe II] $1.64 \mu \mathrm{m}$ line luminosity, assuming Fe gas abundance from Podio et al. (2006). ${ }^{b}$ Upper value determined assuming that only $~ 30 \%$ of iron is in gaseous form.

e.g. Nisini et al. 2002). Given that the considered lines do not cover a wide range of wavelengths, the method is not very sensitive to small $A_{V}$ variations. We find that the best alignment is obtained with $A_{V}$ values between 10 and $20 \mathrm{mag}$. With this range of extinctions, we get $\mathrm{H}_{2}$ column densities ranging between 5 and $13 \times 10^{18} \mathrm{~cm}^{-2}$, corresponding to a mass flux ranging from $\sim 2 \times 10^{-8}$ to $5.3 \times 10^{-8} M_{\odot} \mathrm{yr}^{-1}$. The (one-sided) estimates of $\dot{M}_{\text {loss }}$ we thus derived are summarised in Table 8 .

\section{Discussion}

It is interesting to analyse the derived accretion and ejection properties and their mutual connection in the light of the results provided by some works that have recently investigated the characteristics of embedded protostellar sources. In particular, we compare the results presented here to observations carried out by Nisini et al. (2005a, hereafter N05) on a small sample of Class I sources in the R CrA star-forming region, using the same instrument and observational setup. The most interesting sources (namely HH100-IR and IRS 2) displayed strong H I emission lines along with well-detected absorption features from which stellar properties and veiling could be derived. Unlike the objects analysed in this paper, all the CrA sources showed no signature of jet emission in the region closely surrounding the central star; only HH100-IR has been suggested to be the driving source of the Herbig Haro objects HH101 and HH99 (Hartigan \& Graham 1987) and of a bipolar molecular outflow (Anglada et al. 1989), which are, however, observed far from the central object. To help the comparison, we added in Tables 7 and 8 the parameters of the three CrA sources found by N05 to have H I emission.

We also refer to two recent surveys of embedded sources that investigated the nature of Class I sources with respect to the Class II objects, in order to verify the paradigm of the star formation according to which Class I are actively accreting objects less evolved than Class II: White \& Hillenbrand (2004, hereafter W04) analysed high-resolution optical spectra of a large sample of environmentally young stars (i.e. Class I and Class II objects powering Herbig-Haro flows) in Taurus-Auriga. However, since the Class Is observed by the authors are optically visible, their sample is probably biased toward more evolved objects than standard Class I sources. Doppmann et al. (2005, hereafter D05) studied high-resolution near-IR spectra of a large set of Class I and flat-spectrum sources in various nearby star-forming clouds and derived that Class Is show in average higher veilings and angular velocities than what is observed in Class II sources, as expected for less evolved and accreting objects.

\subsection{Spectral features}

The medium-resolution spectra of our sources show different significant characteristics. In fact, both the detected emission features and their relative strength vary depending on the objects, as can be seen in Figs. 1-3. The $\mathrm{HI}, \mathrm{CO}$, and $\mathrm{H}_{2}$ emission is detected in each of three sources, which in general should be suggestive of the presence of accretion/ejection flows and of an accretion disc. These features are usually, but not always, observed in embedded sources; in particular, D05 detected HI, CO, and $\mathrm{H}_{2}$ emission in $65 \%, 15 \%$ and $44 \%$ of the objects of their sample, respectively, noting that the presence of the $\mathrm{CO}$ is always accompanied by $\mathrm{HI}$ emission. This is a behaviour we find in our sources as well, as in those of N05. In contrast, the presence of permitted lines does not appear to be strictly connected with the simultaneous presence of jet lines from the region closely surrounding the central source. For instance, the $\mathrm{NaI}$ is detected both in HH26 IRS and HH34 IRS, but the [Fe II] lines are only visible in the second source. This lack of correlation is extreme in the case of the CrA objects in N05, with strong permitted emission lines and no jet signatures at all. Therefore, ejection and accretion signatures that are detected in the spectra do not seem to be strictly related.

All these findings must denote different physical conditions in the close surroundings of the sources: such an indication is also given by the analysis of the flux ratio between $\mathrm{Br} \gamma$ and $\mathrm{Na}$ I, which is not constant in the objects (see Table 8). We note that this ratio is smaller in the jet sources with respect to the CrA objects; since $\mathrm{H}$ and $\mathrm{Na}$ have very different ionisation potentials (13.6 and $5.1 \mathrm{eV}$, respectively), a variation of the flux ratio must reflect a different ionisation degree in the region traced by the $\mathrm{Br} \gamma$ and $\mathrm{Na}$ I.

The Na I lines are in most cases observed in Class I sources where $\mathrm{CO}$ emission is detected: 5 objects out of 8 in D05 and 2 out of 2 in N05. In contrast, in the majority of T Tauri stars of D05, both CO and Na I are observed in absorption. Noticeably, in our sample the $\mathrm{CO}(2-0) / \mathrm{Na}$ r ratio (Table 8) follows a trend opposite to the $\mathrm{Br} \gamma / \mathrm{Na}$ I ratio, i.e. it is higher in the jet sources. The $\mathrm{CO}$ overtone emission is usually interpreted as coming from the inner gaseous disc, heated at temperatures of $2000 \mathrm{~K}$ or more by viscous accretion or magnetohydrodynamic waves (e.g. Carr et al. 1993). Sodium I lines could then be originated in an even hotter and internal disc region at low ionisation. We note that in both HH26 IRS and HH34 IRS, the FWHM velocities of the $\mathrm{Na} I$ lines are lower than those of $\mathrm{Br} \gamma$ by a factor of about two. This is evidence that $\mathrm{Br} \gamma$ is associated to a gas component at a higher Keplerian velocity, e.g. the accretion flow and/or ionised stellar/disc wind. All these findings may suggest that sources 
Table 8. Derived parameters for the observed targets.

\begin{tabular}{lcccccc}
\hline \hline Source & $r_{K}$ & $\begin{array}{c}\mathrm{Br} \gamma E W \\
(\AA)\end{array}$ & $\mathrm{Br} \gamma / \mathrm{NaI}$ & $\mathrm{Br} \gamma / \mathrm{H}_{2}$ & $\mathrm{Br} \gamma / \mathrm{Fe}$ II & $\mathrm{CO}(2-0) / \mathrm{Na}$ I \\
\hline HH26 IRS & $>2$ & -5.0 & 1.8 & 0.3 & $>25$ & 15 \\
HH34 IRS & $>5$ & -8.9 & 2.4 & 1.7 & 0.22 & 11.6 \\
HH46 IRS & $>1$ & -4.8 & $>5.1$ & 5.0 & 0.05 & $>30$ \\
\hline HH100-IR & $6.0 \pm 0.5$ & -7.9 & 6.9 & 93.0 & $\ldots$ & 4.6 \\
IRS2 & $2.9 \pm 0.2$ & -7.0 & 19.2 & 140.0 & $\ldots$ & 3.5 \\
IRS5a & $1.0 \pm 0.1$ & -0.6 & $\ldots$ & $\ldots$ & $\ldots$ & $\ldots$ \\
\hline
\end{tabular}

Notes. Columns show in order: $K$ band veiling, $\mathrm{Br} \gamma$ equivalent width, $\mathrm{Br} \gamma / \mathrm{Na}$ I, $\mathrm{Br} \gamma / \mathrm{H}_{2}, \mathrm{Br} \gamma / \mathrm{Fe}$ II and $\mathrm{CO}(2-0) / \mathrm{Na}$ I flux ratios. The same results for three Class I sources of the R CrA region (Nisini et al. 2005a) are reported for comparison (see text for details).

with energetic jets possess massive discs characterised by high columns of warm non-ionised gas.

Finally, the absence of Fe II emission in HH26 IR points to the presence of a colder molecular jet in this source, as also confirmed by the lower $\mathrm{Br} \gamma / \mathrm{H}_{2}$ ratio observed, which indicates a higher $\mathrm{H}_{2}$ content.

\subsection{Veiling}

The lower limits we set on the $K$ band veiling (see Table 8 ) show that at least two sources have veilings greater than the ones typically observed in the more evolved T Tauri stars, for which $r_{\mathrm{K}} \sim 1-2$ (e.g. Folha \& Emerson 1999; Johns-Krull \& Valenti 2001), in agreement with the values measured on other Class I objects (e.g. Doppmann et al. 2005; Nisini et al. 2005a; Greene \& Lada 1996). High $\left(r_{\mathrm{K}}>2\right)$ values of the $K$-band veiling are indicative of the presence of inner circumstellar dusty envelopes (Greene \& Lada 1996) and have been suggested as a good indicator of high accretion activity in the source, since excess emission should be generated by the infalling matter (e.g. Calvet et al. 1997). D05 measured $K$-band veilings for Class I and flatspectrum sources in Ophiuchus that are significantly higher than those of Class II in the same region and assume this as a sign that Class I stars are actually embedded objects undergoing significant mass accretion. Finally, a positive correlation between amount of veiling and accretion luminosity was found by N05 in the small sample of Class Is in R Cra.

If the $K$-band veiling is indeed due to the accretion excess above the stellar photosphere, then one should expect a correlation between the equivalent widths of accretion tracer lines and the veiling. This kind of correlation has indeed been found for $\mathrm{H} \alpha$ and Ca II lines in T Tauri stars (e.g. Muzerolle et al. 1998b). It is interesting to see if such a correlation also exists for $\mathrm{Br} \gamma$ in the limited sample of our jet sources and CrA sources from N05.

On the assumption that the ratio between the $\mathrm{Br} \gamma$ flux and the $K$ band accretion flux is roughly constant, the equivalent width should indeed only depend on the veiling:

$E W \approx \frac{F_{\mathrm{Br} \gamma}}{F_{K, \mathrm{acc}}} \frac{r_{K}}{1+r_{K}}$,

where it has been supposed that all the $K$ band excess emission is due to accretion $\left(F_{K \text {,exc }} \approx F_{K \text {,acc }}\right)$. From this relationship one should expect the line EW to increase roughly linearly for low $r_{K}$ values, reaching an asymptotic value equal to $F_{\mathrm{Br} \gamma} / F_{K \text {,acc }}$ for high values of $r_{K}$.

Despite the limited number of determinations and the lower limits on $r_{K}$ for the jet sources, such behaviour is approximately recognisable in Fig. 7, where the results for the six considered sources are plotted. In particular, the large $\mathrm{Br} \gamma \mathrm{EW}$ measured on HH34 IRS should be noticed, which is similar to the value

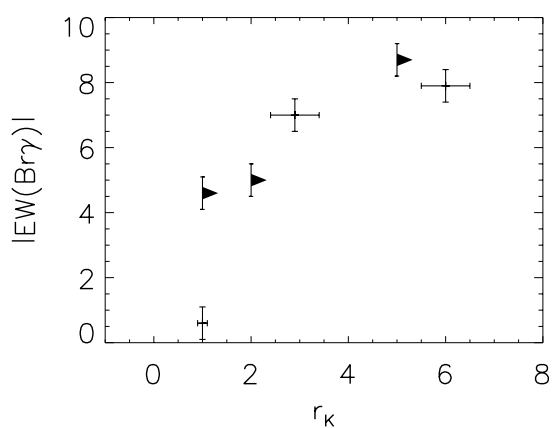

Fig. 7. $\mathrm{Br} \gamma$ equivalent widths is plotted against the $K$ band veiling for the three sources and the CrA sources of Nisini et al. (2005a).

measured on HH100 IR and in agreement with what is expected given the large lower limit on $r_{K}$. This indicates that the accretion luminosity in this object should indeed be high, providing support to our estimate of an $A_{K}$ value of the order of 5 mag.

\subsection{Accretion luminosity}

The sources analysed here have accretion luminosities that are a fraction between $\sim 0.2$ and 0.8 of the total luminosity. In addition to HH34 IRS and HH26 IRS, only three Class I have been found to have a $L_{\text {acc }} / L_{\text {bol }}$ ratio $\gtrsim 0.5$ so far: HH100-IR and IRS2 in CrA (N05) and YLW15 in Oph (Greene \& Lada 2002). We note that the fraction of $L_{\mathrm{acc}}$ over $L_{\mathrm{bol}}$ in HH46 IRS is somehow uncertain, due to the binary nature of the object.

Recent findings have actually shown that a large spread seems to exist in the measured accretion luminosities of embedded sources. Accretion luminosities from a few percent to about $50 \%$ of the total have been measured for instance by W04 in the Taurus-Auriga sources of their sample, although these results (based on optically visible sources) would apply better to objects generally more evolved than our embedded targets. A similar result has also recently been found, however, by Beck (2007) for a sample of Class Is in Taurus. Finally, in N05 only two out of 5 investigated Class Is have bolometric luminosities dominated by accretion. D05 note that the accretion luminosity derived from $\operatorname{Br} \gamma$ fluxes using Muzerolle's relation for the sources of their sample is low $\left(10^{-1}<L_{\mathrm{acc}} / L_{\odot}<10^{0}\right)$ even in objects with significantly high bolometric luminosities, which could therefore be explained only by assuming a very high photospheric contribution. This last result could in part be due to underestimating the extinction correction to be applied to the $\operatorname{Br} \gamma$, being the $A_{V}$ value estimated from near-IR colours. There is nevertheless growing evidence that only a limited number of sources normally classified as Class I actually have accretion-dominated luminosity. 
On the basis of the small sample we have gathered, we can try to tentatively define some properties characterising these accretion-dominated young objects (ADYOs), i.e. those sources with $L_{\mathrm{acc}} / L_{\mathrm{bol}} \gtrsim 0.5$. First of all, we note that all the identified accretion-dominated objects have $r_{K}$ strictly larger than 2 . In the D05 sample, only 6 out of 40 sources have such a large veiling, showing that ADYOs might be just a fraction of all the Class Is. Sources with massive and dense jets detected very close to the exciting object have high $L_{\mathrm{acc}} / L_{\mathrm{bol}}$ ratios, as we infer from the sample investigated in this paper, but the presence of a jet is not a necessary characteristic of an ADYO (e.g. CrA IRS 2). On the other hand, the fact that the jet sources investigated here have SEDs typical of objects in transitions from Class 0 to Class I indicates that large accretion luminosities are associated to less evolved sources, while a consistent number of "classical" Class I objects may be in a phase of less intense accretion and have already acquired most of their final mass.

We also note that all the ADYOs discovered so far, except for YLW15, have CO and $\mathrm{Na} I$ lines in emission, although there is no direct correlation between the strength of these features and the accretion luminosity. The majority of the Class Is investigated in D05, as well as in Beck (2007), show on the contrary CO and $\mathrm{Na}$ I features in absorption, which is another indication that their samples may be biased towards sources lacking massive accretion-heated inner discs.

\subsection{Mass accretion and loss rates}

The actual mechanism through which accretion and ejection of matter take place in the protostar still remains substantially unknown from an observational point of view. Measurements of the accretion and ejection parameters are therefore very important for providing the observational constraints needed to disentangle the proposed models. A quantity of interest is the ratio $\dot{M}_{\text {loss }} / \dot{M}_{\text {acc }}$, whose expected value is in general of the order of 0.01-0.1 in disc wind models (e.g. Ferreira 1997; Casse \& Ferreira 2000), while a (two-sided) value of 0.3 is needed in the X-wind scenario of Shu et al. (1994).

In our case, the measured loss rates of $10^{-8}-10^{-7} M_{\odot} \mathrm{yr}^{-1}$ are comprised in the range of values that have been observed for other Class I and Class II sources (e.g. Podio et al. 2006; Gullbring et al. 1998; White \& Hillenbrand 2004), while the observed accretion rate range of $10^{-7}-10^{-6} M_{\odot} \mathrm{yr}^{-1}$ (derived from the $\mathrm{H}$ I line luminosity) extends above the values found for the most actively accreting Class II jet sources (Mohanty et al. 2005).

From these measurements we derive a mass flux that is $\sim 0.01$ of the accretion rate in HH26 IRS and in HH34 IRS, which is in good agreement with values derived in T Tauri stars and with models, while for HH46 IRS a higher value $>0.1$ is inferred (see Table 7).

Of course, the reliability of these values depends on the precision with which $\dot{M}_{\text {loss }}$ and $\dot{M}_{\text {acc }}$ are determined. These quantities might be affected, for example, by systematic errors due to the methods used to derive them. For instance, mass flux rates measured from optical or near-IR line luminosities may be underestimated in the presence of a dense and cold jet neutral component, which is not traced by the considered lines. Similarly, mass accretion rates estimates are affected by the intrinsic scatter of the Muzerolle's relation and by the stellar parameters inferred when assuming the source on the birthline.

Nevertheless, the observed spread could also be explained, at least in part, in terms of variable accretion and ejection rates of the sources. The accretion process (and consequently the related ejection of matter) is in fact likely to undergo phases of burst and quiescence (see e.g. Larson 1984), similar to what has been suggested for other kind of accreting astronomical objects. In this scenario, Class I sources would display phases of enhanced accretion with a corresponding increase in the total luminosity. Evidence in this sense has been provided for few Class I sources (e.g. SSV13, Liseau et al. 1992), and the existence of accretion/ejection phases is clearly observed in the jets themselves, where various distinct knots of emission are visible, often at the same distance from the source, in the red and blue lobes.

We note that variability of the accretion can produce systematic biases when comparing the rates. This is because the estimates of the mass flow can only be made on the jet knot closer to the protostar and therefore are derived observing matter that was ejected before the accretion process ongoing at the moment of observations, whose signatures we detect on the spectrum. In this case, a better estimate would take into account an accretion rate averaged over the time during which the matter responsible for the jet emission has been ejected, rather than the instantaneous accretion rate measured from the spectra.

Unfortunately, the values of the $\dot{M}_{\text {loss }} / \dot{M}_{\text {acc }}$ ratio are probably affected by this kind of problem in a significant number of cases.

\section{Conclusions}

We have investigated the accretion and ejection properties of three embedded sources (HH26 IRS, HH34 IRS, HH46 IRS) showing prominent jet-like structures. To this aim we analysed their medium-resolution $(R \sim 9000)$ near-IR spectra acquired with VLT-ISAAC. The main results we obtained can be summarised as follows:

- The bolometric luminosity and SEDs of the three sources have been revised on the basis of Spitzer and recent sub-mm observations and of the theoretical radiative transfer models available in the literature. It turns out that the sources are probably very young, in a transition phase between Class 0 and I.

- The spectra of the three sources show significant differences in their characteristics: in fact, the number and the absolute and relative intensity of the observed emission features (associated both with the accretion region and the jet environment) vary among the sources. In particular, we point out that there is no clear relationship between the presence of the jet and the spectral accretion signatures detected. Moreover, the spectra show no sign of absorption features, indicating a large amount of veiling that in turn suggests the presence of warm dusty envelopes around the sources, heated by the active ongoing accretion.

- In two of our sources (HH34 IRS and HH26 IRS), we find a $\mathrm{Br} \gamma / \mathrm{Na}$ I ratio much higher (by a factor of three or more) than observed in other Class Is or T Tauri stars. Conversely, the ratio between the $\mathrm{CO}(2-0) 2.3 \mu \mathrm{m}$ overtone emission and the $\mathrm{Na}$ I $2.20 \mu \mathrm{m}$ doublet shows the opposite trend, i.e. is lower in the objects with jets. This may indicate the presence of massive discs around the jet sources, characterised by large amounts of warm gas in a neutral state.

- We consistently derive $A_{K}, L_{*}$, and $L_{\text {acc }}$ assuming the three sources on the birthline and adopting the relationship between $\operatorname{Br} \gamma$ luminosity and accretion luminosity derived by Muzerolle et al. (1998a). In the case of HH34 IRS and HH26 IRS, accretion largely contributes to the total energy, as expected for young sources in the main accretion phase. In HH46 IRS, we estimate an $L_{\text {acc }} / L_{\text {bol }}$ ratio of the order 
of 0.2 , but the real value largely depends on how the bolometric luminosity is shared among the two binary components. Taking into account $K$-band veiling values $r_{K}$ greater than the lower limits inferred from the spectra would generally lead to higher $A_{K}, L_{\text {acc }}$, and $\dot{M}_{\text {acc }}$.

- Mass accretion and loss rates span (including errors) the range $10^{-8}-10^{-6} M_{\odot} \mathrm{yr}^{-1}$. The derived $\dot{M}_{\text {loss }} / \dot{M}_{\text {acc }}$ ratio is $\sim 0.01-0.03$ for HH26 IRS and HH34 IRS and >0.1 for HH46 IRS. These numbers lie within the range of values usually predicted by models and found in the more active classical T Tauri stars.

- Comparing the results found in this work with other spectroscopic studies recently performed on Class I sources, we conclude that the number of Class Is actually having accretion-dominated luminosities (accretion-dominated young objects, ADYOs) could be limited. From the properties inferred in a small sample of objects, we can tentatively define some criteria to characterise such sources: ADYOs have all $K$-band veiling larger than 2 and, in the majority of the cases, present (in addition to $\mathrm{HI}$ ) IR features of $\mathrm{CO}$ and $\mathrm{NaI}$ in emission, although these do not directly correlate with the accretion luminosity. Class Is with massive jets have high $L_{\mathrm{acc}} / L_{\mathrm{bol}}$ ratios, but not all the identified ADYOs present a jet. The SEDs of our small sample of three objects suggest that accretion-dominated sources could be in an evolutionary phase in transition between Class 0 and I. Of course, studies of the kind presented here, but carried out on larger samples of possible candidates, should be performed in order to test and refine the tentative criteria that we have just mentioned.

Acknowledgements. We are grateful to the referee Sylvie Cabrit, whose insightful comments and suggestions helped us to improve the paper. This work was partially supported by the European Community's Marie Curie Research and Training Network JETSET (Jet Simulations, Experiment and Thory) under contract MRTN-CT-2004-005592. This publication makes use of data products from the Two Micron All Sky Survey, which is a joint project of the University of Massachusetts and the Infrared Processing and Analysis Center/California Institute of Technology, funded by the National Aeronautics and Space Administration and the National Science Foundation. This work is based in part on observations made with the Spitzer Space Telescope, which is operated by the Jet Propulsion Laboratory, California Institute of Technology under a contract with NASA.

\section{References}

André, P., Ward-Thompson, D., \& Barsony, M. 1993, ApJ, 406, 122

Anglada, G., Rodriguez, L. F., Torrelles, J. M., et al. 1989, ApJ, 341, 208

Anglada, G., Estalella, R., Mauersberger, R., et al. 1995, ApJ, 443, 682

Asplund, M., Grevesse, N., \& Sauval, A. J. 2005, in Cosmic Abundances as Records of Stellar Evolution and Nucleosynthesis, ed. T. G. Barnes, III, \& F. N. Bash, ASP Conf. Ser., 336, 25

Bacciotti, F., \& Eislöffel, J. 1999, A\&A, 342, 717

Bally, J., \& Devine, D. 1994, ApJ, 428, L65

Beck, T. L. 2007, AJ, 133, 1673

Boogert, A. C. A., Pontoppidan, K. M., Lahuis, F., et al. 2004, ApJS, 154, 359

Calvet, N., Hartmann, L., \& Strom, S. E. 1997, ApJ, 481, 912

Calzoletti, L., Giannini, T., Nisini, B., \& Lorenzetti, D. 2007, A\&A, in preparation

Carr, J. S. 1989, ApJ, 345, 522

Carr, J. S., Tokunaga, A. T., Najita, J., Shu, F. H., \& Glassgold, A. E. 1993, ApJ, 411, L37

Casse, F., \& Ferreira, J. 2000, A\&A, 353, 1115

Chernin, L. M., \& Masson, C. R. 1991, ApJ, 382, L93

Chiar, J. E., Ennico, K., Pendleton, Y. J., et al. 2007, ApJ, 666, L73

Chrysostomou, A., Bacciotti, F., Nisini, B., et al. 2007, A\&A, in preparation

Cohen, M., \& Schwartz, R. D. 1987, ApJ, 316, 311

Correia, J. C., Griffin, M. J., Saraceno, P., \& Molinari, S. 1998, in Star Formation with the Infrared Space Observatory, ed. J. Yun, \& L. Liseau, ASP Conf. Ser., 132,249
Davis, C. J., Ray, T. P., Eisloeffel, J., \& Corcoran, D. 1997, A\&A, 324, 263 Davis, C. J., Ray, T. P., Desroches, L., \& Aspin, C. 2001, MNRAS, 326, 524 Davis, C. J., Stern, L., Ray, T. P., \& Chrysostomou, A. 2002, A\&A, 382, 1021 Doppmann, G. W., Greene, T. P., Covey, K. R., \& Lada, C. J. 2005, AJ, 130, 1145

Dougados, C., Cabrit, S., Lavalley, C., \& Ménard, F. 2000, A\&A, 357, L61

Eislöffel, J., \& Mundt, R. 1992, A\&A, 263, 292

Eislöffel, J., \& Mundt, R. 1994, A\&A, 284, 530

Eislöffel, J., Davis, C. J., Ray, T. P., \& Mundt, R. 1994, ApJ, 422, L91

Fernandes, A. J. L. 2000, MNRAS, 315, 657

Ferreira, J. 1997, A\&A, 319, 340

Ferreira, J., Dougados, C., \& Cabrit, S. 2006, A\&A, 453, 785

Folha, D. F. M., \& Emerson, J. P. 1999, A\&A, 352, 517

Garcia Lopez, R., Nisini, B., Giannini, T., \& Lorenzetti, D. 2007, A\&A, in preparation

Giannini, T., McCoey, C., Caratti o Garatti, A., et al. 2004, A\&A, 419, 999

Gibb, A. G., Little, L. T., Heaton, B. D., \& Lehtinen, K. K. 1995, MNRAS, 277, 341

Greene, T. P., \& Lada, C. J. 1996, AJ, 112, 2184

Greene, T. P., \& Lada, C. J. 2002, AJ, 124, 2185

Gullbring, E., Hartmann, L., Briceno, C., \& Calvet, N. 1998, ApJ, 492, 323

Hartigan, P., \& Graham, J. A. 1987, AJ, 93, 913

Hartigan, P., Morse, J. A., \& Raymond, J. 1994, ApJ, 436, 125

Hartigan, P., Edwards, S., \& Ghandour, L. 1995, ApJ, 452, 736

Heathcote, S., \& Reipurth, B. 1992, AJ, 104, 2193

Johns-Krull, C. M., \& Valenti, J. A. 2001, ApJ, 561, 1060

Johnstone, D., \& Bally, J. 2006, ApJ, in press

Johnstone, D., Fich, M., Mitchell, G. F., \& Moriarty-Schieven, G. 2001, ApJ, 559,307

Johnstone, D., Matthews, H., \& Mitchell, G. F. 2006, ApJ, 639, 259

Königl, A., \& Pudritz, R. E. 2000, Protostars and Planets IV, 759

Larson, R. B. 1984, MNRAS, 206, 197

Lis, D. C., Menten, K. M., \& Zylka, R. 1999, ApJ, 527, 856

Liseau, R., Lorenzetti, D., \& Molinari, S. 1992, A\&A, 253, 119

Massi, F., Giannini, T., Lorenzetti, D., et al. 1999, A\&AS, 136, 471

Mathis, J. S. 1998, ApJ, 497, 824

Meyer, M. R., Calvet, N., \& Hillenbrand, L. A. 1997, AJ, 114, 288

Mohanty, S., Jayawardhana, R., \& Basri, G. 2005, ApJ, 626, 498

Muzerolle, J., Hartmann, L., \& Calvet, N. 1998a, AJ, 116, 2965

Muzerolle, J., Hartmann, L., \& Calvet, N. 1998b, AJ, 116, 455

Najita, J., Carr, J. S., Glassgold, A. E., Shu, F. H., \& Tokunaga, A. T. 1996, ApJ, 462, 919

Nisini, B., Caratti o Garatti, A., Giannini, T., \& Lorenzetti, D. 2002, A\&A, 393, 1035

Nisini, B., Antoniucci, S., Giannini, T., \& Lorenzetti, D. 2005a, A\&A, 429, 543

Nisini, B., Bacciotti, F., Giannini, T., et al. 2005b, A\&A, 441, 159

Noriega-Crespo, A., Morris, P., Marleau, F. R., et al. 2004, ApJS, 154, 352

Palla, F., \& Stahler, S. W. 1993, ApJ, 418, 414

Podio, L., Bacciotti, F., Nisini, B., et al. 2006, A\&A, 456, 189

Quinet, P., Le Dourneuf, M., \& Zeippen, C. J. 1996, A\&AS, 120, 361

Rebull, L. M., Stapelfeldt, K. R., Evans, II, N. J., et al. 2007, ArXiv Astrophysics e-prints

Reipurth, B., Bally, J., Graham, J. A., Lane, A. P., \& Zealey, W. J. 1986, A\&A, 164,51

Reipurth, B., Chini, R., Krugel, E., Kreysa, E., \& Sievers, A. 1993, A\&A, 273, 221

Reipurth, B., Yu, K. C., Heathcote, S., Bally, J., \& Rodríguez, L. F. 2000, AJ, 120,1449

Reipurth, B., Heathcote, S., Morse, J., Hartigan, P., \& Bally, J. 2002, AJ, 123, 362

Rieke, G. H., \& Lebofsky, M. J. 1985, ApJ, 288, 618

Robitaille, T. P., Whitney, B. A., Indebetouw, R., Wood, K., \& Denzmore, P. 2006, ApJS, 167, 256

Rodríguez, L. F., \& Reipurth, B. 1996, Rev. Mex. Astron. Astrofis., 32, 27

Shu, F., Najita, J., Ostriker, E., et al. 1994, ApJ, 429, 781

Siess, L., Dufour, E., \& Forestini, M. 2000, A\&A, 358, 593

Simon, T., Andrews, S. M., Rayner, J. T., \& Drake, S. A. 2004, ApJ, 611, 940

Skrutskie, M. F., Cutri, R. M., Stiening, R., et al. 2006, AJ, 131, 1163

Stark, D. P., Whitney, B. A., Stassun, K., \& Wood, K. 2006, ApJ, 649, 900

Takami, M., Chrysostomou, A., Ray, T. P., et al. 2006, ApJ, 641, 357

Tanaka, M., Sato, S., Nagata, T., \& Yamamoto, T. 1990, ApJ, 352, 724

Wallace, L., \& Hinkle, K. 1997, ApJS, 111, 445

White, R. J., \& Hillenbrand, L. A. 2004, ApJ, 616, 998

Whitney, B. A., Wood, K., Bjorkman, J. E., \& Wolff, M. J. 2003, ApJ, 591, 1049

Woitas, J., Bacciotti, F., Ray, T. P., et al. 2005, A\&A, 432, 149 